\begin{tabular}{lcc}
\hline Ročník LIII & 18 & Ć́slo 4, 2005 \\
\hline
\end{tabular}

\title{
CATALOGUE OF ALIEN ANIMAL SPECIES IN THE CZECH REPUBLIC
}

\author{
H. Šefrová, Z. Laštůvka
}

Received: June 10, 2005

\begin{abstract}
ŠEFROVÁ, H., LAŠTƯVKA, Z.: Catalogue of alien animal species in the Czech Republic. Acta univ. agric. et silvic. Mendel. Brun., 2005, LIII, No. 4, pp. 151-170

The catalogue of alien animal species registered in the Czech Republic, with data on their origin, date on the first observation, way of introduction (accidental, deliberate, spontaneous), invasive status (casual, non-invasive, post-invasive, invasive), habitat (eusynanthropic, urban, agricultural, natural), trophic requirements and possible influences (plant or stored-product pest, biodiversity influence). In total 595 species are listed, i.e. $1.8 \%$ of the fauna of this country; of these, 22 species of molluscs $(8.8 \%$ of the local fauna), $451 \mathrm{spp}$. of arthropods (1.5\%), $383 \mathrm{spp}$. of insects (1.4\%), and $55 \mathrm{spp}$. of vertebrates (9.2\%). Among the registered species, $248 \mathrm{spp}$. (41.8\%) are confined to closed and heated spaces by their occurrence, and $287 \mathrm{spp}$. have become naturalized (48.2\%). Of these $113 \mathrm{spp}$. are considered invasive (19\% of alien spp.). 65 spp. (10.9\% of aliens) are pests of stored products, 84 spp. (14.1\%) are parasites of important animals, 53 spp. (8.9\%) are pests of plants grown in heated rooms (above all, glasshouses), $28 \mathrm{ssp} .(4.7 \%)$ are agricultural or forest pests, and 39 spp. (6.6\%) may influence local biodiversity. The origin of the naturalized alien species is mostly in North America (70;24.4\%), the Mediterranean (61; $21.3 \%)$, E Asia (44; 15.4\%), Central and SW Asia (43; 15\%), and S or SE Asia (30; 10.5\%).
\end{abstract}

alien and invasive animal species, Czech Republic

For many reasons, alien organisms have become the objects of increasing studies. As a rule, the presence of animals is not as conspicuous as that of plants. Therefore, their invasions may evade the attention of the public, and yet the consequences of their presence in their new environment are analogical. According to their ecological requirements and dispersal capacity, alien animal species are confined by their occurrence to closed and heated spaces only, or their populations can exist in the open. The former are at least unwelcome roommates of man, but much more frequently, these species are molesting, hygienically defective, destructive to various materials, or pests of room and glasshouse plants. The latter group of alien animals, coming from climatically similar regions, either occur in anthropically disturbed or controlled communities and can become pests of agricultural crops or parasi- tes of free-living animals, or they can invade semi-natural or natural communities and more or less importantly affect their genetic as well as species diversity (by changing competitive relationships, predation, herbivory, disturbance and changes on trophic chains, habitat modification, introduction of non-indigenous diseases and parasites, etc.).

Introduced or invasive animal species were studied as early as the second half of the 19th century (cf. e.g. Krüger, 1899) and more intensely from the 1950s onwards (e.g. Elton, 1958). The biological invasions, their causes, forms, and consequences as regards central Europe have recently been assessed by Kowarik (2003); the economic costs associated with alien and invasive species of various organisms and in various regions of the world have been evaluated by Pimentel (2002). Comprehensive analyses are available of the 
past invasions, endeavouring to discover the characteristics that are common to the invasive species and finding possible ways of predicting new invasions (cf. e.g. Agassiz, 1996; Pyšek, 2001; Kowarik, 2003). Alien animal species have received due attention even in central Europe; for instance, some 500 animal species of alien origin, 300 of which became established, have been reported from Austria (Essl \& Rabitsch, 2002); 1100 species, incl. some 280 established ones, from Germany (Geiter et al., 2002). As to the Czech Republic, there are a catalogue containing alien plant species (Pyšek et al., 2002) and many papers dealing with individual alien animals, but no comprehensive evidence or list of animal species of alien origin is available as yet.

\section{Definition of terms used}

The terms "alien" and especially "invasive" have not been understood or used quite uniformly (cf. Richardson et al., 2000). Thus, the term "invasive" has occasionally been applied to denote those alien species that cause aesthetic or economic damage; in other cases, it has only been applied to those species that invade semi-natural and natural communities and affect the local biodiversity (cf. e.g. Manchester \& Bullock, 2000; Davis \& Thompson, 2001; or Convention on Biological Diversity). In either case, no attention has been paid to the essential criterion - the invasive dispersal of such species. The above problems have been discussed in detail and the pertaining terms have been clearly defined e.g. by Richardson et al. (2000), Pyšek et al. (2002) and Richardson \& Pyšek (2004). Without any major difficulties, the terminology proposed by these authors can be applied to all groups of organisms, and we have adopted it in the present catalogue. Despite the unequivocal definition of the particular groups, the biological and ecological specific characters of animals (vagility, more complex, and diversified habitat links, position at various levels of the trophic chain, etc.) may cause greater difficulties in classifying them than in the case of plants.

The term "alien" is applied to all animal species which naturally do not occur in a particular area and have got in it through direct or indirect activity of man and can produce in it at least short-term populations uncontrolled by man. Such species were introduced either deliberately or accidentally, spontaneously spreading in urban habitats, or accompanying an introduced host (plant or animal). The term "alien" does not apply to species having spontaneously dispersed from their native ranges (expansive species, Pyšek et al., 2002), those introduced through natural mechanisms, above all, birds, nor those invading central Europe due to various causes for short periods, periodically or non-periodically (seasonal or longer invasions of certain species, above all, insects and birds, mi- grating species, etc.). Nor does this term cover those species which, while spreading spontaneously, their expansion may have been made possible by far-reaching landscape modifications (Richardson \& Pyšek, 2004), excepting unequivocal man-made cases (previous introduction of host species, exclusively synanthropic occurrence).

According to the duration and character of occurrence, the category of alien species is divided into:

1. Eusynanthropic species - S, which, after having been introduced, survive for only a short period and reproduce only inside buildings, greenhouses and other heated objects and gradually vanish or are liquidated (as indicated by a letter in brackets), or produce in such places long-term populations and possibly are dispersed by man (partly even spontaneously during the warm period of the year); exceptionally, some of such species can produce short-term populations in the open - SC.

2. Casual alien species $-\mathrm{C}$, which, having escaped or been released in the open, will only produce short-term populations and vanish again (this group not including individuals having escaped from captivity, or individual captures of introduced animals that did not further reproduce).

3. Naturalized, non-invasive species $-\mathrm{N}$, producing long-term populations in the external environment, populating suitable habitats in the place of introduction, not dispersing or, if so, then only over the immediate vicinity of the place.

4. Naturalized, post-invasive species (Pyšek et al., 2002) populated the territory in the past and are known, since the beginning of their study, as fully naturalized, more or less without any changes in their distribution - *N, e.g. Aceria tristriata, $A$. erinea, Caloptilia roscipennella, Chromaphis juglandicola and Panaphis juglandis (all trophically tied to Juglans regia). These species evidently invaded central Europe prior to 1900 and there is no way of finding out whether they were dispersed with their host plant, followed it immediately, or invaded the territory with some time delay. Apparently, the invasions of many species took place long after their host species had been introduced, which at the same time suggests that the dispersal of the host may not be the only factor to initiate invasion in several cases. For instance, the invasion of the gracillariid moth, Phyllonorycter platani, did not take place until during the 20th century (Šefrová, 2001) and that of the lygaeid bug, Arocatus longiceps, only in the late of the century (Stehlík \& Hradil, 2000) although plane trees (Platanus spp.), their host species, have been planted in central Europe for roughly 200 years. These species are considered invasive and are included in 
the next group. A somewhat peculiar group consists of several fish and bird species which, while not reproducing naturally, are often kept in the open, sometimes in considerable numbers, and can thus distinctly affect processes in seminatural and natural ecosystems as well as their biodiversity $-\mathrm{M}$.

5. Invasive species $-\mathrm{I}$, representing that part of the naturalized species which will not stay in the place of introduction but will disperse from it, populating territories of varying sizes. The rate of such animal invasions is sometimes distinctly higher than that of plant species (cf. Richardson et al., 2000), usually attaining kilometres, or even hundreds of kilometres a year. For example, the present invasion of Cameraria ohridella in Europe proceeds at a rate of some $50 \mathrm{~km}$ a year (Šefrová, 2003).

The category of invasive species has a slightly different connotation in the case of animal parasites introduced together with their hosts. Here only those species are considered invasive which can invade additional host species and disperse with them. Those parasitic species that stay with their initial hosts are classified as naturalized, non-invasive, even though they can further disperse together with their hosts; cf. e.g., two fluke species introduced into Europe from North America (Erhardová-Kotrlá, 1971; Tenora, 1956). Fascioloides magna, introduced with Odocoileus virginianus, can infest other cervid species and is considered an invasive species, whereas Quinqueserialis quinqueserialis stays with its original host species, Ondatra zibethicus, it does not infest autochthonous species, and cannot affect native biodiversity. Therefore, it is included in the category of naturalized, non-invasive species.

The occurrence of naturalized species is limited to urban habitats (U) to which they are tied in terms of climate, structure of the habitat, or food (feeding on decorative plants, domestic animals, and other synanthropic species), or they disperse over the landscape by occupying habitats created by man (agricultural crops, purpose-made vegetation, man-made aquatic habitats, ruderal areas) (R), or by invading semi-natural and natural communities (A). The animal and several plant parasites are connected close with their hosts, their occurrence is dependent on the host occurrence more than on the habitat character $(\mathrm{H})$.

\section{Questionable cases and excluded species}

The catalogue does not comprise alien species kept in households, aquariums, terrariums, zoological gardens and in similar enclosed breedings (enclosures, parks), the same as laboratory species including their specific parasites. Domesticated animal species, not autochthonous in central Europe and not occurring there in feral forms, and their specific parasites, are not included either; in the Czech Republic, such species include Cairinia moschata (Linnaeus, 1758), Gallus gallus (Linnaeus, 1758), Pavo cristatus Linnaeus, 1758, Numida meleagris Linnaeus, 1758, and/ or Cavia tschudii Fitziger, 1857. None of such species can produce populations not controlled by man, and they do not influence (or infest) native animal species. Not considered are cases of introduced individuals of alien species that did not subsequently reproduce (e.g. Černý, 1985), nor unsuccessful attempts at intentional introductions (e.g. Frank, 1970; cf. also Baruš \& Oliva, 1995).

In central Europe, the ranges of many animal species are permanently shifting at various rates and various extent, and in most cases the natural changes are difficult to distinguish from those caused by anthropogenic influences. The natural shifts took place both in the pre-Neolithic and in the present period. Therefore, one can hardly accept the criterion by which the species that colonized the territory after the Neolithic should be considered "alien" (cf. Webb, 1985; Manchester \& Bullock, 2000). In contrast to plants, most animals usually leave no long-term traces of their presence (such as pollen, non-decomposed remains, skeletons, shells). In evaluating their historic occurrence, therefore, their study (but for rare exceptions) is limited to a very short period, i.e. roughly from the mid-19th century onwards (cf. Lauterer, 2004). Even if one could determine more closely the date of their earlier arrival in central Europe, one cannot decide whether their dispersal was spontaneous, conditioned by anthropic factors, or directly introduced by man. That is especially true of numerous species of arthropods inhabiting treeless regions, many of which probably could not exist in central Europe without the extensive agricultural activity and other anthropic landscape modifications. Therefore, we take it unnecessary to separate, for the animals, the "archeozoa" category analogical to the plant category of "archeophyta". The few cases of animal species introduced prior to 1500 should better be included in the group of "alien species" or added to that of "neozoa" (cf. Kowarik, 2003).

The catalogue includes phytophagous species accompanying on their specific, deliberately introduced host plants (both archeo- and neophytes), but the species with trophic relations to accidentally or spontaneously spreading archeophytes are omit, similarly as the species living on plant species which could be (?) native in a part of the territory (e.g. Cytisus scoparius or Vitis vinifera). Similarly, those species which are autochthonous in at least a part of the country and have been deliberately or involuntarily introduced into many other places are not considered here as "alien". 


\section{Conception of the catalogue}

Besides a simple enumeration of species, the catalogue also contains further items of information useful from the economic, ecological, and environmental points of view. Within their respective higher taxa, the species are given in an alphabetical order. Because of their expected use especially in the Czech Republic, the scientific names of species are accompanied by their Czech vernacular ones. As far as known, the origin of the species is stated; often the native ranges are unknown in the cases of species of considerable or even cosmopolitan distribution, dispersed over considerably large areas prior to 1900 . Dates are given of the first records of the occurrence in the Czech Republic (insufficiently known in several cases). In this point, there may be considerable disproportions between particular species. In the cases of deliberate and documented introductions, of important pests or medialized species the date actually corresponds with that of the first occurrence or the beginning invasion. In a number of species, however, the date of the first record may not agree at all with that of the introduction and dispersal. It may refer, for example, to the first damage registered, or to the period in which the respective taxonomic group was studied for the first time. In such cases, the date is marked with an asterisk *. Most probably, specialized animal and several plant parasites were first introduced together with their host, but this may have taken place during subsequent introductions as well. That is why also in such cases the date of the first faunistic observation, if available, is given and marked with the asterisk. Tentative data on the trophic requirements suggest the participation of an alien species in ecosystems (synanthropic, urban, agricultural, semi-natural to natural) and its possible economic as well as ecological (parasitological, hygienic) influence. Invariably the most natural habitat invaded by the alien species is stated. The column headed "Source" contains references on published particulars on a given species (date of introduction, bionomics, influences, etc.). Where possible, comprehensive publications are cited, comprising references to partial items of information, not necessarily the first paper published on that particular species.

\section{Results: situation in the Czech Republic}

The territory of the Czech Republic is known to be inhabited by about 34000 species (own actual data) of metazoan animals. To-date, 595 species of alien origin have been registered, i.e. $1.8 \%$ of the total. Of the introduced species, 248 are confined in closed heated spaces (eusynanthropic species, 41.8\%), 53 of them being pests of home and glasshouse plants, and 65 are pests of stored products. Another 60 species $(10.1 \%)$ can survive in the open for only a short pe- riod and hence their practical or ecological influence is nil as a rule. The remaining 287 naturalized species (48.2\% of aliens) can survive in the open for long periods. Of these, $105 \mathrm{spp}$. (17.7\% of aliens) will stay in the place of introduction (they are non-invasive), 69 spp. (11.6\% of aliens) dispersed in the past (post-invasive), and 113 spp. (19\% of aliens) can be considered invasive (Tab. I, Fig. 1). Of the 113 invasive species, 7 are limited to urban habitats, 15 inhabit the cultivated landscape (both productive and uncontrolled communities), $58 \mathrm{spp}$. invade even semi-natural to natural habitats, and $33 \mathrm{spp}$. are distributed with their host, independently of the habitat; 84 species $(14.1 \%$ of aliens) are parasites of important animals, 28 naturalized species $(4.7 \%)$ are pests of agricultural crops and forestry, and 39 (6.6\%) can potentially or actually affect the local biodiversity (Fig. 2).

The above overall classification of alien species will slightly differ when individual higher taxa are evaluated. In particular, major differences are found between invertebrates and vertebrates. Objectively, they are due to their size, bionomics, and ecological position of the two groups; subjectively, to the greater volume of information on the vertebrate species as well as to the fact that unsuccessful introductions of invertebrates are not registered as a rule. Of the 55 species of introduced vertebrates, only three are eusynanthropic (5.5\%), but 22 species (40\%) may affect biodiversity. At the same time, biodiversity can even be affected by species of occasional occurrence as well as by the naturalized non-invasive ones. Hence, the number of species that can possibly affect biodiversity is several times higher than of the invasive ones. On the other hand, of the 540 introduced invertebrate species $245 \mathrm{spp}$. (45.5\%) are eusynanthropic, and only $17 \mathrm{spp}$. (3.2\%) can affect biodiversity; all of them are invasive but represent only a small fraction of the invasive species. The situation in selected important taxonomic groups is shown in Tab. I.

Likewise, there are differences in different taxonomic groups as regards the ways and motivation of introduction. On the whole, accidentally introduced species make up the overwhelming majority of cases $(80 \%)$. About $11 \%$ of species were introduced deliberately (game and decorative species, fishes, species utilised in biological control), and the remaining $9 \%$ followed spontaneously their introduced host plants. As to vertebrates, only about $31 \%$ were introduced accidentally, $65 \%$ deliberately, and about $4 \%$ dispersed spontaneously. As to invertebrates, accidental introduction pertains to almost $85 \%$ of species, a mere $5 \%$ being introduced deliberately, and about $10 \%$ spontaneously spread over our territory (Fig. 3).

The origin of the alien species is diverse, and unknown in a number of cases. Many invertebrate species (Coccinea, Lepidoptera, Coleoptera) were found and 
described for the first time in the places of their introduction. Most of the eusynanthropic species come from the tropics and subtropics. The naturalized species were mostly introduced from North America (70; $24.4 \%$ ), the Mediterranean (61;21.3\%), E Asia (44; $15.4 \%)$, Central and SW Asia (43; 15\%), and S or SE Asia $(30 ; 10.5 \%)$. Concrete data on the origin of the naturalized species are given in Fig. 4.

Although there is no general way of predicting which additional species could be introduced in Europe the possible immigrants can be estimated at least from the situation in the neighbouring countries.
Thus, for example, one can expect the occurrence in the Czech Republic of Urnatella gracilis Leidy, 1851 (Entoprocta), Stephanitis takeyai Drake \& Maa, 1955 (Heteroptera), or Gnathotrichus materiarius (Fitch, 1858), Xylosandrus germanus (Blandford, 1894), Neoclytus acuminatus (Fabricius, 1775) (Coleoptera), and other insect species. Some of the introduced and invasive species may be present in the region for a long time but may not have been registered for various reasons, e.g. some of the Monogenea, or several species of mites of the families Tenuipalpidae and Eriophyidae.

I: Composition and numbers of alien animal species in the Czech Republic (important taxa selected); $S$-eusynanthropic, $C$-casual, $N$-naturalized, non-invasive, $* N$-post-invasive, $I$-invasive

\begin{tabular}{|l|r|r|r|r|r|r|r|r|}
\hline Taxon & Spp. CR & Aliens & \% & S & C & N & *N & I \\
\hline Plathelminthes & 670 & 29 & 4.3 & 1 & - & 12 & - & 16 \\
\hline Nematoda & 950 & 32 & 3.4 & 5 & 1 & 15 & 8 & 3 \\
\hline Mollusca & 246 & 22 & 8.9 & 9 & 2 & 1 & - & 10 \\
\hline Araneida & 851 & 22 & 2.6 & 18 & 3 & - & - & 1 \\
\hline Insecta & 27200 & 383 & 1.4 & 200 & 28 & 36 & 50 & 69 \\
\hline Arthropoda (total) & 30500 & 451 & 1.5 & 230 & 34 & 53 & 58 & 76 \\
\hline Invertebrata (total) & 33400 & 540 & 1.6 & 245 & 37 & 85 & 66 & 107 \\
\hline Vertebrata & 595 & 55 & 9.2 & 3 & 23 & 20 & 3 & 6 \\
\hline Total & $\mathbf{3 4 \mathbf { 0 0 0 }}$ & $\mathbf{5 9 5}$ & $\mathbf{1 . 8}$ & $\mathbf{2 4 8}$ & $\mathbf{6 0}$ & $\mathbf{1 0 5}$ & $\mathbf{6 9}$ & $\mathbf{1 1 3}$ \\
\hline
\end{tabular}

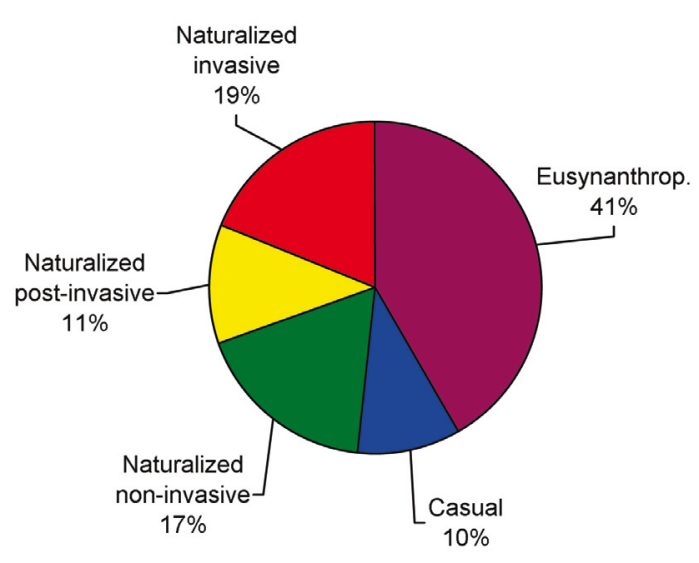

1: Categories of alien animal species

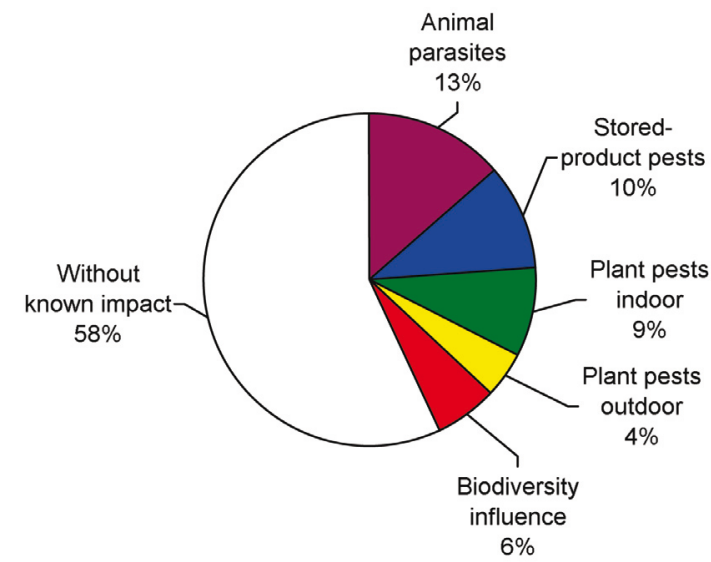

2: Impact of alien species 

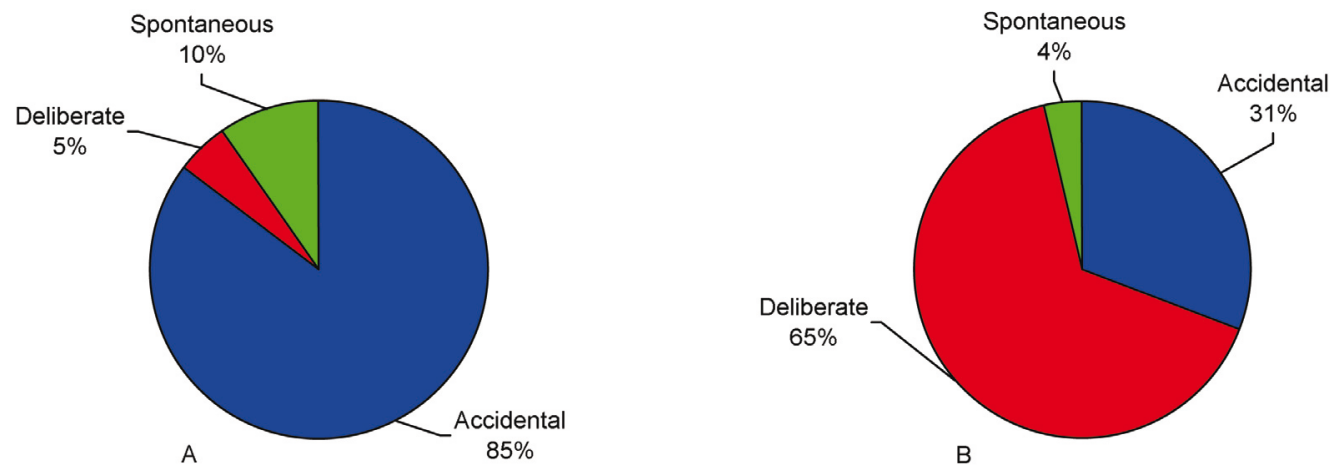

3: Motivation and form of introduction; $A$-Invertebrata, $B$-Vertebrata

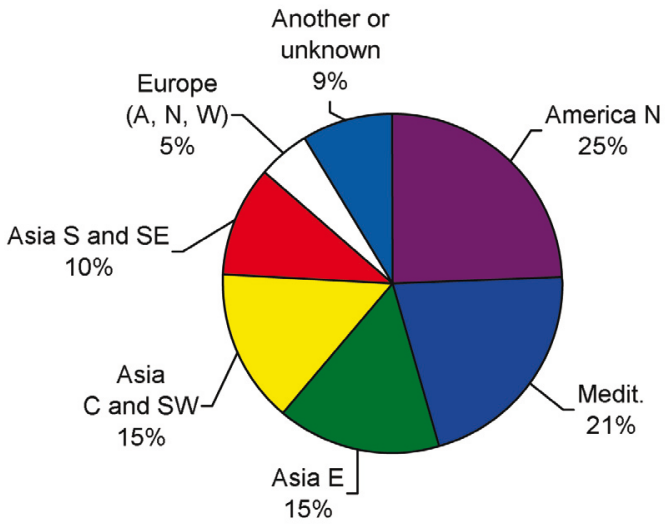

4: Origin of naturalized alien species

Appendix I. Catalogue of alien animal species in the Czech Republic.

Origin: AF - Africa, AM - America, AS - Asia, AUS - Australia, EU - Europe, ME - Mediterranean, NZE - New Zealand, TR - Pan(sub)tropical, A - Alps, C - central, E - east, N - north, S - south, W - west; Status of alien species and their occurrence: $\mathrm{C}$ - casual, $\mathrm{I}$ - invasive, $\mathrm{M}$ - breeding (in the open), artificial stripping (in fishes), $\mathrm{N}$ - naturalized, non-invasive, ${ }^{*} \mathrm{~N}$ - naturalized, post-invasive, $\mathrm{S}$ - eusynanthropic only, (S) - occasional eusynanthropic occurrence (short-time occurrence after introduction); habitats (the second letter): A - seminatural or natural, $\mathrm{H}$ - occurring with its host, $\mathrm{R}$ - cultivated landscape (agricultural habitats, ornamental cultures, ruderals, etc.), U - settlements (urban habitats, relation to the urban environment, to ornamental plants, or to domestic animals), $\uparrow-$ probably extinct; 1st: Date of the introduction, or of the first occurrence, *date - the first observation (usually not the first occurrence), or the first publication occasionally; Introduction: A - accidental, D - deliberate, S - spontaneous; Food (trophic relation): $\mathrm{Pa}$ - zooparasitic, parasitoid, $\mathrm{Ph}$ - phytophagous (phylophagous), Pl - planktonophagous, Po - polyphagous, $\mathrm{Pr}$ - predatory, Sa - saprophagous, Xy - xylophagous; impact (second letter in brackets): $\mathrm{P}$ - plant pest, $\mathrm{S}$ - stored-product pest, B - possibility of biodiversity influence; Source: number of a reference. 


\begin{tabular}{|c|c|c|c|c|c|c|c|}
\hline Taxon & Czech name & Origin & Stat & 1st & Intr & Food & Source \\
\hline Plathelminthes: Turbellaria & Ploštěnky & & & & & & \\
\hline Dugesia tigrina (Girard, 1850) & ploštěnka americká & AMN & $\mathrm{IA}$ & 1935 & A & $\mathrm{Pr}$ & 79 \\
\hline Plathelminthes: Monogenea & Jednorodí & & & & & & \\
\hline Cleidodiscus pricei Mueller, 1936 & žábrohlíst & AMN & $\mathrm{NH}$ & *1970 & A & Pa: Ictalurus & 37 \\
\hline Dactylogyrus achmerowi Gusev, 1955 & žábrohlíst & ASE & IA & *1988 & A & Pa: Cyprinus & 54,155 \\
\hline Dactylogyrus inexpectatus Izjumova, 1955 & žábrohlíst & ASE & $\mathrm{IA}$ & *1999 & A & Pa: Carassius auratus & 155,167 \\
\hline Dactylogyrus lamellatus Achmerow, 1952 & žábrohlíst & ASE & $\mathrm{NH}$ & *1980 & A & Pa: Ctenopharyngodon & 155 \\
\hline Dactylogyrus nobilis Long \& Yu, 1958 & žábrohlíst & ASE & $\mathrm{NH}$ & *1980 & A & Pa: Aristichthys & 155 \\
\hline Dactylogyrus squameus Gusev, 1955 & žábrohlíst & ASE & $\mathrm{NH}$ & *1998 & A & Pa: Pseudorasbora & 168 \\
\hline Eudiplozoon nipponicum (Goto, 1891) & žábrohlíst & ASE & IA & *1986 & A & $\mathrm{Pa}(\mathrm{B})$ : Cyprinus & 52,155 \\
\hline Gyrodactylus cyprini Diarova, 1964 & žábrohlíst & ASE & IA & *1970 & A & Pa: Cyprinus, Abramis & 37 \\
\hline Gyrodactylus sprostonae Ling, 1962 & žábrohlíst & ASE & IA & *1991 & A & $\mathrm{Pa}(\mathrm{B})$ : Cyprinus, Carassius & 53 \\
\hline Pseudodactylogyrus anguillae (Yin \& Sproston, 1948) & žábrohlíst & ASE & IA & *1994 & A & Pa: Anguilla & 155,234 \\
\hline Pseudodactylogyrus bini (Kikuchi, 1929) & žábrohlíst & ASE & IA & *1994 & A & Pa: Anguilla & 155,234 \\
\hline Plathelminthes: Trematoda & Motolice & & & & & & \\
\hline Fascioloides magna (Bassi, 1875) & motolice obrovská & AMN & $\mathrm{IA}$ & *1927 & A & $\mathrm{Pa}(\mathrm{B})$ : Cervidae & 41 \\
\hline Quinqueserialis quinqueserialis Bark. \& Laug., 1911 & motolice & AMN & $\mathrm{NH}$ & 1905 & A & Pa: Ondatra & 239 \\
\hline Plathelminthes: Cestoda & Tasemnice & & & & & & \\
\hline Andrya cuniculi (Blanchard, 1891) & tasemnice & MEW & $\mathrm{NH}$ & *1958 & A & Pa: Oryctolagus & 39 \\
\hline Echinolepis carioca (Magelhaes, 1898) & tasemnice & ASS & $\mathrm{NH}$ & *1957 & A & Pa: Gallus, Meleagris & 237 \\
\hline Khawia sinensis Hsü, 1935 & tasemnice & ASE & IA & 1960 & A & $\mathrm{Pa}(\mathrm{B})$ : Cyprinus & 186 \\
\hline Moniezia rupicaprae Galli-Valerio, 1929 & tasemnice & EUA & $\mathrm{NH}$ & *2004 & A & Pa: Rupicapra & 241 \\
\hline Mosgovoyia ctenoides (Railliet, 1890) & tasemnice & MEW & $\mathrm{NH}$ & *1956 & $A$ & Pa: Oryctolagus & 242 \\
\hline Proteocephalus longicollis (Zeder, 1800) & tasemnice & ?AMN & IA & 1977 & A & Pa: Salmonidae & 155 \\
\hline Blatticola blattae (Graeffe, 1860) & roup & $?$ & $\mathrm{SH}$ & *1955 & A & Pa: Blattidae & 56 \\
\hline Cactodera cacti (Filipjev \& Stekhoven, 1941) & hád’átko & AMC & $\mathrm{S}$ & 1971 & A & $\mathrm{Ph}(\mathrm{P})$ : Cactaceae & 201 \\
\hline Cooperia curticei (Giles, 1892) & vlasovka drobná & ASSW & *NA & *1948 & A & Pa: Caprinae & 100,200 \\
\hline Cystocaulus ocreatus Railliet \& Henry, 1907 & plicnivka & ASSW & $\mathrm{NH}$ & *1957 & A & Pa: Caprinae & 38 \\
\hline Dictyocaulus filaria (Rudolphi, 1809) & průduchovka ovčí & ASSW & $\mathrm{NH}$ & *1950 & A & Pa: Ovis & 200 \\
\hline Eucoleus gastricus (Baylis, 1926) & kapilárie & ASSE & $\mathrm{NH}$ & *1959 & $A$ & Pa: Rattus & 40 \\
\hline Globodera pallida (Stone, 1973) & hád'átko světlé & AMS & $\mathrm{NH}$ & 2002 & A & $\mathrm{Ph}(\mathrm{P})$ : Solanum & 264 \\
\hline Globodera rostochiensis (Wollenweber, 1923) & hád'átko bramborové & AMS & $\mathrm{NH}$ & *1954 & A & $\mathrm{Ph}(\mathrm{P})$ : Solanum & 201 \\
\hline Gongylonema neoplasticum (Fibiger \& Ditlev., 1914) & spirura & ASSE & $\mathrm{NH}$ & *1942 & A & Pa: Rattus & 40,102 \\
\hline Hammerschmidtiella diesingi (Hammersch., 1838) & roup & $?$ & $\mathrm{SH}$ & *1955 & A & Pa: Blattidae & 56 \\
\hline Heterodera fici Kirjanova, 1954 & hád’átko & ? & S & 1975 & A & $\mathrm{Ph}(\mathrm{P})$ : Ficus & 201 \\
\hline Leidynema appendiculatum (Leidy, 1850) & roup & AMC & $\mathrm{SH}$ & *1955 & A & Pa: Blattidae & 56 \\
\hline Marshallagia marshalli (Ransom, 1907) & vlasovka & ASSW & ${ }^{*} \mathrm{NA}$ & *1950 & A & Pa: Ovis, Capra & 200 \\
\hline Muellerius capillaris (Müller, 1889) & plicnivka ovčí & ASSW & ${ }^{*} \mathrm{NA}$ & *1948 & A & Pa: Caprinae & 100 \\
\hline Muellerius tenuispiculatus Gebauer, 1932 & plicnivka & EUA & $\mathrm{NH}$ & *1955 & A & Pa: Rupicapra & 38 \\
\hline Neostrongylus linearis (Marotel, 1913) & plicnivka & ASSW & ${ }^{*} \mathrm{NA}$ & *1956 & A & Pa: Caprinae & 38 \\
\hline Oesophagostomum columbianum Curtice, 1890 & zubovka jelení & ASSW & *NA & *1950 & A & Pa: Caprinae & 200 \\
\hline Ostertagia mossi Dikmans, 1931 & vlasovka & AMN & $\mathrm{NH}$ & 1884 & $A$ & Pa: Odocoileus & 119 \\
\hline Protostrongylus rufescens (Leucart, 1865) & plicnivka & ASSW & ${ }^{*} \mathrm{NA}$ & *1948 & A & Pa: Caprinae & 100,200 \\
\hline Spiculopteragia asymmetrica (Ware, 1925) & vlasovka & ASSW & ${ }^{*} \mathrm{NA}$ & *1970 & A & $\mathrm{Pa}(\mathrm{B})$ : Cervidae & 119 \\
\hline Strongyloides myopotami Artigas \& Pacheco, 1933 & hádě & AMS & $\mathrm{NH}$ & *1952 & A & Pa: Myocastor & 260 \\
\hline Strongyloides ratti Sandground, 1925 & hádě & ASS & ${ }^{*} \mathrm{NH}$ & *1955 & A & Pa: Rattus, Myocastor & 39 \\
\hline Strongyloides stercoralis (Bavay, 1876) & hádě střevní & TR & C & *1948 & A & Pa: (Homo) & 101 \\
\hline Syphacia muris (Yamaguti, 1935) & roup & ASS & $\mathrm{NH}$ & *1960 & A & Pa: Rattus & 243 \\
\hline Trichosomoides crassicauda (Bellingham, 1840) & svalovec & ASS & $\mathrm{NH}$ & *1958 & A & Pa: Rattus & 39 \\
\hline
\end{tabular}




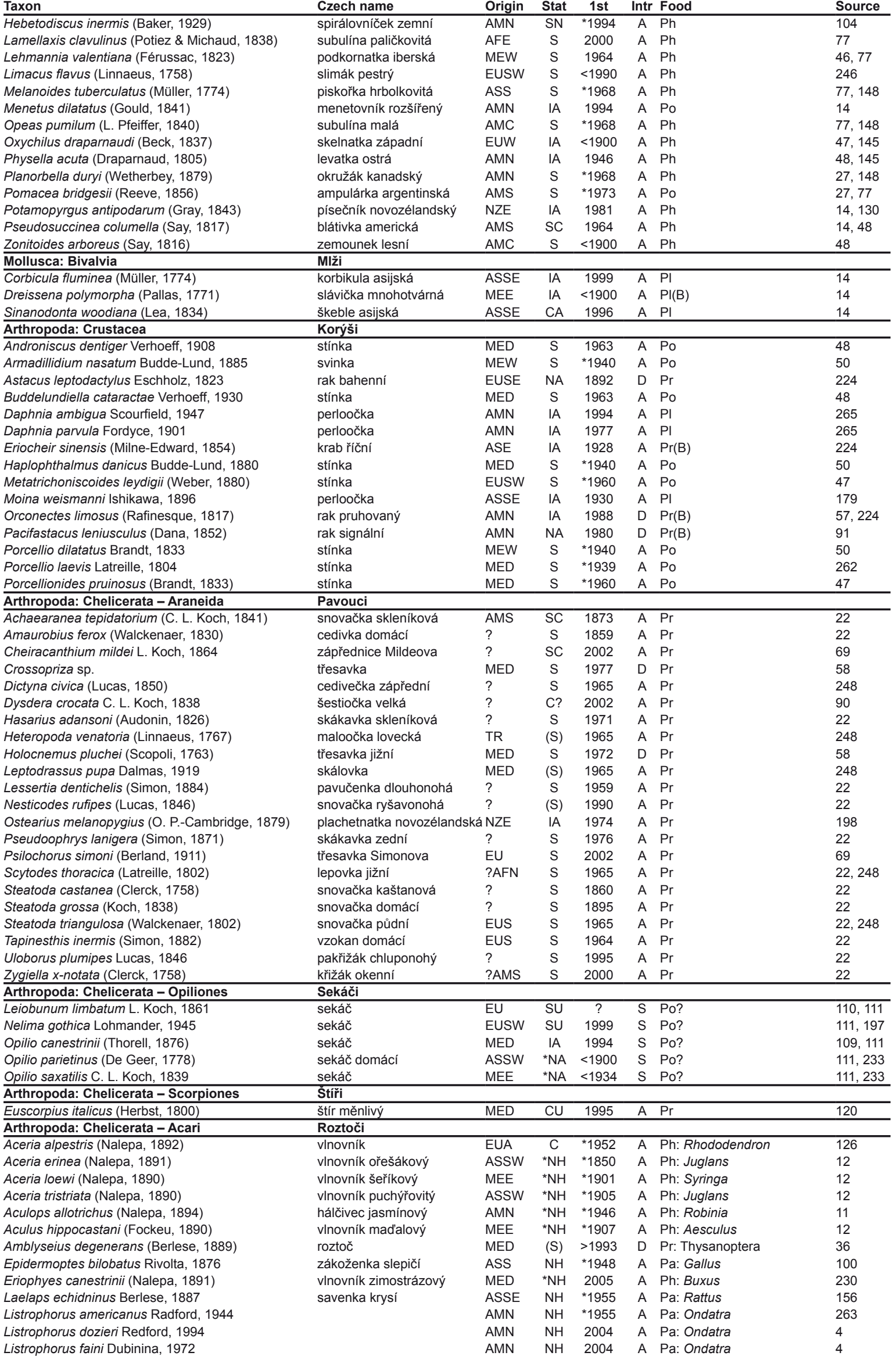


Taxon

Czech name

Origin Stat 1st Intr Food

Listrophorus gibbus Pagenstecher, 1862

Listrophorus validus Banks, 1910

Myocoptes ondatrae Lukoschus \& Rouwet, 1968

Neoseiulus californicus (McGregor, 1954)

Ondatralaelaps multispinosus (Banks, 1909)

Ornithonyssus bacoti (Hirst, 1913)

Phyllocoptes azaleae Nalepa, 1904

Phytoseiulus persimilis Athias-Henriot, 1957

Psoroptes cuniculi (Delafond, 1859)

Trixacarus diversus Sellnick, 1944

Varroa destructor Anderson \& Trueman, 2000

Arthropoda: Myriapoda - Diplopoda

Orthomorpha gracilis (C. L. Koch, 1847)

Arthropoda: Insecta - Zygentoma

Lepisma saccharina Linnaeus, 1758

Thermobia domestica (Packard, 1873)

Arthropoda: Insecta - Orthoptera

Acheta domestica (Linnaeus, 1758)

Diestrammena asynamora (Adelung, 1902)

Troglophillus neglectus (Kraus, 1879) Arthropoda: Insecta - Blattodea

Blatta orientalis Linnaeus, 1758

Blattella germanica (Linnaeus, 1767)

Nauphoeta cinerea (Olivier, 1789)

Neostylopyga rhombifolia (Ståll, 1861)

Periplaneta americana (Linnaeus, 1758)

Periplaneta australasiae (Fabricius, 1775)

Periplaneta brunnea Burmeister, 1838

Pycnoscelus surinamensis (Linaeus, 1767)

Supella longipalpa (Fabricius, 1798)

Arthropoda: Insecta - Dermaptera Euborellia annulipes (Dohrn, 1864)

Arthropoda: Insecta - Heteroptera

Amphiareus obscuriceps (Poppius, 1909)

Anthocoris butleri Le Quesne, 1954

Arocatus longiceps Stål, 1872

Corythucha ciliata (Say, 1832)

Deraeocoris flavilinea (A. Costa, 1862)

Elasmotropis testacea (Herrich-Schäffer, 1830)

Macrolophus glaucescens Fieber, 1858

Macrolophus melanotoma (A. Costa, 1853)

Orius insidiosus (Say, 1832)

Orsillus depressus (Mulsant \& Rey, 1852)

Oxycarenus lavaterae (Fabricius, 1787)

Stephanitis rhododendri Horváth, 1905

Tuponia elegans (Jakovlev, 1867)

Tuponia hippophaes (Fieber, 1861)

Xylocoris flavipes (Reuter, 1875)

Arthropoda: Insecta - Auchenorrhyncha

Endria nebulosa (Ball, 1900)

Eupteryx melissae Curtis, 1837

Graphocephala fennahi Young, 1977

Japananus hyalinus (Osborn, 1900)

Macropsis elaeagni Emeljanov, 1964

Metcalfa pruinosa (Say, 1830)

Opsius stactogalus Fieber, 1866

Paradorydium paradoxum (Her.-Sch., 1837)

Stictocephala bisonia Kopp \& Yonke, 1977

Arthropoda: Insecta - Psyllinea

Psylla buxi (Linnaeus, 1758)

Cacopsylla hippophaes (Förster, 1848)

Cacopsylla zetterstedti (Thomson, 1877)

Calophya rhois (Löw, 1877)

Livilla variegata (Löw, 1881)

Trioza alacris Flor, 1861

Epitrioza neglecta Loginova, 1977

Arthropoda: Insecta - Aleyrodinea

Bemisia tabaci (Gennadius, 1889)

Dialeurodes chittendeni Laing, 1928

Trialeurodes lauri (Signoret, 1882)

Trialeurodes vaporariorum (Westwood, 1856)

Arthropoda: Insecta - Aphidinea

Acyrthosiphon caraganae (Cholodkovsky, 1908)

Acyrthosiphon ignotum Mordvilko, 1914

Aphis forbesi Weed, 1889

Aphis gossypii Glover, 1877

Aphis spiraephaga Müller, 1961

Aphrastasia pectinatae (Cholodkowsky, 1888)

Appendiseta robiniae (Gillette, 1907)

\begin{tabular}{|c|c|c|c|c|c|c|}
\hline Czech name & Origin & Stat & $1 \mathrm{st}$ & Intr & Food & Source \\
\hline & MEW & $\mathrm{NH}$ & *1943 & A & Pa: Oryctolagus & 122,263 \\
\hline & AMN & $\mathrm{NH}$ & 2004 & $A$ & Pa: Ondatra & 4 \\
\hline & AMN & $\mathrm{NH}$ & 2004 & $A$ & Pa: Ondatra & 4 \\
\hline roztoč & AMN & $(\mathrm{S})$ & $>1995$ & $\mathrm{D}$ & Pr: Tetranychidae & 36 \\
\hline savenka ondatří & AMN & $\mathrm{NH}$ & *1955 & $A$ & Pa: Ondatra & 156 \\
\hline čmelík & ASSE & $\mathrm{NH}$ & 1952 & $A$ & Pa: Rattus & 156 \\
\hline hálčivec azalkový & ASE & $S$ & *1952 & A & Ph: Rhododendron & 126 \\
\hline roztoč & AMS? & C & 1974 & $\mathrm{D}$ & Pr: Tetranychidae & 93,157 \\
\hline prašivka králičí & MEW & $\mathrm{NH}$ & *1948 & A & Pa: (Oryctolagus) & 100 \\
\hline zákožka & $?$ & $\mathrm{NH}$ & $?$ & $A$ & Pa: Rattus & 195 \\
\hline roztoč včelí & ASS & $\mathrm{NH}$ & 1981 & $\mathrm{~A}$ & Pa: Apis & 187 \\
\hline \multicolumn{7}{|l|}{ Mnohonožky } \\
\hline plochule skleníková & ASSE & $\mathrm{S}$ & $<1950$ & $\mathrm{~A}$ & $\mathrm{Sa}$ & 131 \\
\hline \multicolumn{7}{|l|}{ Śupinušky } \\
\hline rybenka domácí & MED & $\bar{S}$ & ${ }^{*} 1890$ & $\bar{A}$ & $\mathrm{Sa}$ & 125 \\
\hline rybenka skleníková & MED & S & *1945 & $\mathrm{A}$ & $\mathrm{Sa}$ & 125 \\
\hline \multicolumn{7}{|l|}{ Rovnokřídlí } \\
\hline cvrček domácí & MED & $\bar{S}$ & $<1900$ & $A$ & $\mathrm{Po}(\mathrm{S})$ & 162 \\
\hline koník skleníkový & ASE & S & 1891 & $A$ & Po & 75 \\
\hline koník jeskynní & MED & NR & 1998 & A & Po & 76 \\
\hline \multicolumn{7}{|l|}{ Švábi } \\
\hline šváb obecný & ASS & $\mathrm{S}$ & $<1850$ & $\mathrm{~A}$ & $\mathrm{Po}(\mathrm{S})$ & 162 \\
\hline rus domácí & ASS & S & $<1850$ & A & $\mathrm{Po}(\mathrm{S})$ & 162 \\
\hline šváb šedý & AMC & $(\mathrm{S})$ & $?$ & A & Po & 121, 195 \\
\hline šváb harlekýn & $?$ & (S) & $?$ & $A$ & Po & 121 \\
\hline šváb americký & AMC & $S$ & 1920 & $A$ & $\mathrm{Po}(\mathrm{S})$ & 162 \\
\hline šváb australský & ?AUS & $(\mathrm{S})$ & 1950 & $A$ & Po & 126 \\
\hline šváb hnědý & $?$ & (S) & 1992 & $A$ & Po & 117,218 \\
\hline šváb surinamský & AMS & $(\mathrm{S})$ & 1950 & $A$ & Po & 121,126 \\
\hline šváb hnědopruhý & $\mathrm{AFC}$ & (S) & 1975 & $\mathrm{~A}$ & Po & 199 \\
\hline \multicolumn{7}{|l|}{ škvoři } \\
\hline škvor jižní & ?MED & $\mathrm{C}$ & 1912 & $\mathrm{~A}$ & $\mathrm{Po}$ & 87 \\
\hline \multicolumn{7}{|l|}{ Ploštice } \\
\hline hladěnka východní & ASE & $\mathrm{IA}$ & *1994 & $\bar{S}$ & $\mathrm{Pr}$ & 113 \\
\hline hladěnka zimostrázová & EUSW & ${ }^{*} \mathrm{NU}$ & *1962 & S & Pr: Buxus & 214 \\
\hline ploštička platanová & MEE & $\mathrm{IH}$ & 1998 & $S$ & Ph: Platanus & 216 \\
\hline sítnatka platanová & AMN & $\mathrm{IH}$ & 1995 & A & $\mathrm{Ph}(\mathrm{P})$ : Platanus & 215 \\
\hline klopuška italská & MEW & $\mathrm{IR}$ & 2003 & $S$ & $\operatorname{Pr}$ & 113 \\
\hline sit'natka bělotrnová & MEE & ${ }^{*} \mathrm{NA}$ & $<1844$ & $\mathrm{~S}$ & Ph: Echinops & 44 \\
\hline klopuška bělotrnová & EUSE & ${ }^{*} \mathrm{NA}$ & $<1858$ & S & Ph: Echinops & 45 \\
\hline klopuška skleníková & MED & $(\mathrm{S})$ & $>1990$ & $\mathrm{D}$ & Pr: Aleyrodidae & 36 \\
\hline hladěnka skleníková & AMN & (S) & 1992 & $\mathrm{D}$ & Pr: (Thysanoptera) & 68 \\
\hline ploštička cypřišová & MED & ${ }^{*} \mathrm{NR}$ & *1994 & $S$ & Ph: Cupressaceae & 217 \\
\hline ploštička lipová & MEW & IR & 2004 & $S$ & Ph: Tilia (Malvaceae) & 114 \\
\hline sítnatka pěnišníková & ASE & ${ }^{*} \mathrm{NR}$ & *1941 & $A$ & Ph: Rhododendron & 238 \\
\hline klopuška půvabná & ASCSW & ${ }^{*} \mathrm{NH}$ & *1971 & $S$ & Ph: Tamarix & 21 \\
\hline klopuška tamaryšková & MED & ${ }^{*} \mathrm{NH}$ & ${ }^{*} 2001$ & $S$ & Ph: Tamarix & 21 \\
\hline hladěnka skladištní & $?$ & $(\mathrm{~S})$ & 1966 & $\mathrm{~A}$ & $\operatorname{Pr}$ & 254 \\
\hline Kř́ísi & & & & & & \\
\hline křísek & ?AMN & ${ }^{*} \mathrm{NA}$ & ${ }^{*} 1977$ & $A$ & Ph: Poaceae & 31 \\
\hline pidikřísek & MED & IU & 1955 & $\mathrm{~S}$ & Ph: Lamiaceae & 139 \\
\hline sítinovka pěnišníková & AMN & $\mathrm{IH}$ & 2004 & A & Ph: Rhododendron & 235 \\
\hline křisek & ASE & IA & 1979 & $A$ & $\mathrm{Ph}:$ Acer & 135 \\
\hline křísek & ASC & ${ }^{*} \mathrm{NH}$ & *1982 & $S$ & Ph: Elaeagnus & 137 \\
\hline voskovka zavlečená & AMN & $\mathrm{CU}$ & 2001 & A & $\mathrm{Ph}$ & 141 \\
\hline křísek & MED & $\mathrm{IH}$ & 1953 & $S$ & $\mathrm{Ph}$ : Tamarix & 29 \\
\hline křísek & MED & $\mathrm{C}$ & 1928 & $A$ & $\mathrm{Ph}$ & 163 \\
\hline ostnohřbetka ovocná & AMN & $\mathrm{IA}$ & 1994 & $\mathrm{~A}$ & $\mathrm{Ph}$ & 140 \\
\hline Mery & & & & & & \\
\hline mera zimostrázová & MEW & ${ }^{*} \mathrm{NH}$ & $<1900$ & $\mathrm{~S}$ & Ph: Buxus & 33 \\
\hline mera & EUAN & ${ }^{*} \mathrm{NH}$ & *1969 & $S$ & Ph: Hippophae & 136 \\
\hline mera & EUAN & ${ }^{*} \mathrm{NH}$ & *1980 & $\mathrm{S}$ & Ph: Hippophae & 136 \\
\hline mera škumpová & MED & ${ }^{*} \mathrm{NH}$ & 1906 & $A$ & Ph: Cotinus & 12 \\
\hline mera & MED & ${ }^{*} \mathrm{NH}$ & *1999 & $\mathrm{s}$ & Ph: Laburnum & 143 \\
\hline merule vavřínová & MED & $(\mathrm{S})$ & 1936 & A & Ph: Laurus & 10 \\
\hline merule & ASC & ${ }^{*} \mathrm{NH}$ & ${ }^{*} 1982$ & $\mathrm{~s}$ & Ph: Elaeagnus & 138 \\
\hline Molice & & & & & & \\
\hline molice bavlníková & MED & $\mathrm{S}$ & 1988 & $\mathrm{~A}$ & $\mathrm{Ph}(\mathrm{P})$ & 258 \\
\hline molice pěnišníková & ?EUA & ${ }^{*} \mathrm{NH}$ & *1959 & $A$ & $\mathrm{Ph}(\mathrm{P}):$ Rhododendron & 256 \\
\hline molice vavřínová & MED & $(\mathrm{S})$ & *1952 & A & Ph: Laurus & 126 \\
\hline molice skleníková & AMS & $\mathrm{S}$ & *1941 & $\mathrm{A}$ & $\mathrm{Ph}(\mathrm{P})$ & 28 \\
\hline Mšice & & & & & & \\
\hline kyjatka & ASC & ${ }^{*} \mathrm{NH}$ & ${ }^{*} 1957$ & $\mathrm{~S}$ & Ph: Caragana & 72 \\
\hline kyjatka & ASC & ${ }^{*} \mathrm{NH}$ & *1958 & $S$ & Ph: Spiraea & 72 \\
\hline mšice & AMN & $\mathrm{IA}$ & *1969 & $A$ & $\mathrm{Ph}(\mathrm{P})$ : Fragaria & 74 \\
\hline mšice bavlníková & ?ASSW & $S$ & $<1959$ & $A$ & $\mathrm{Ph}(\mathrm{P})$ & 183 \\
\hline mšice tavolníková & ASC & ${ }^{*} \mathrm{NH}$ & *1957 & $S$ & $\mathrm{Ph}(\mathrm{P})$ : Spiraea & 72 \\
\hline korovnice & ASN & IA & *1977 & S & Ph: Picea, Abies & 74 \\
\hline zdobnatka akátová & AMN & $\mathrm{IH}$ & *1984 & A & $\mathrm{Ph}:$ Robinia & 72 \\
\hline
\end{tabular}




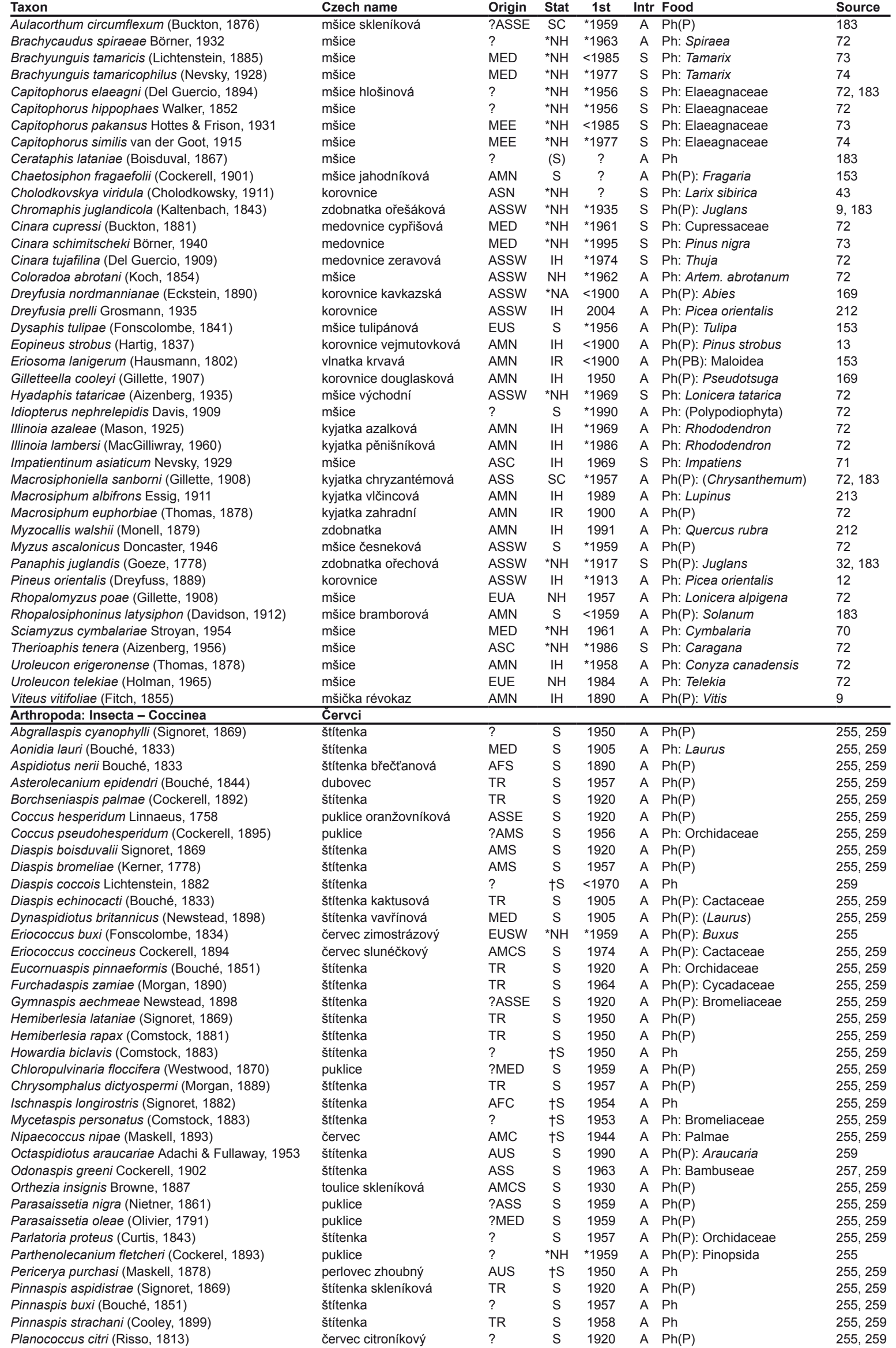




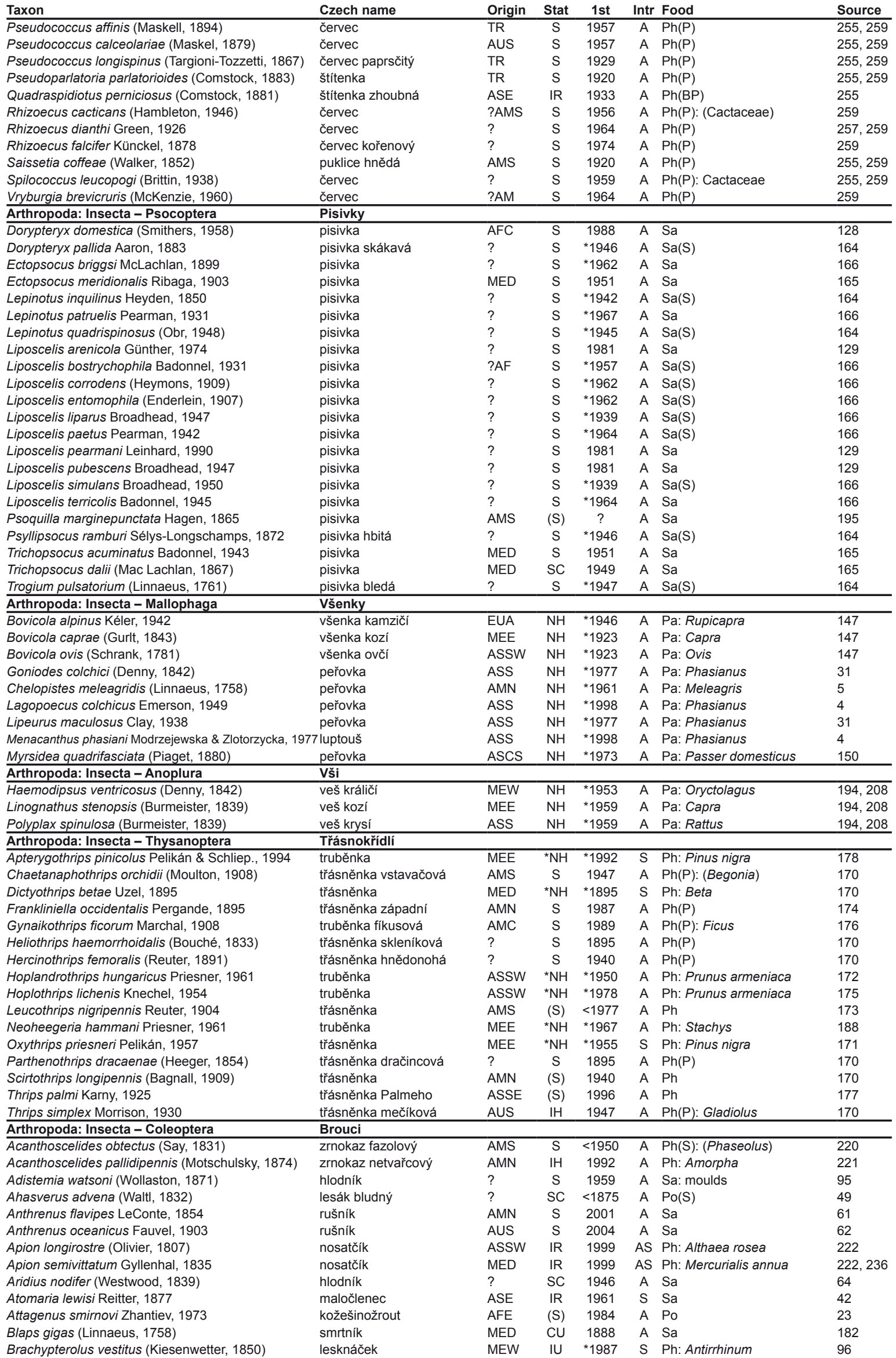




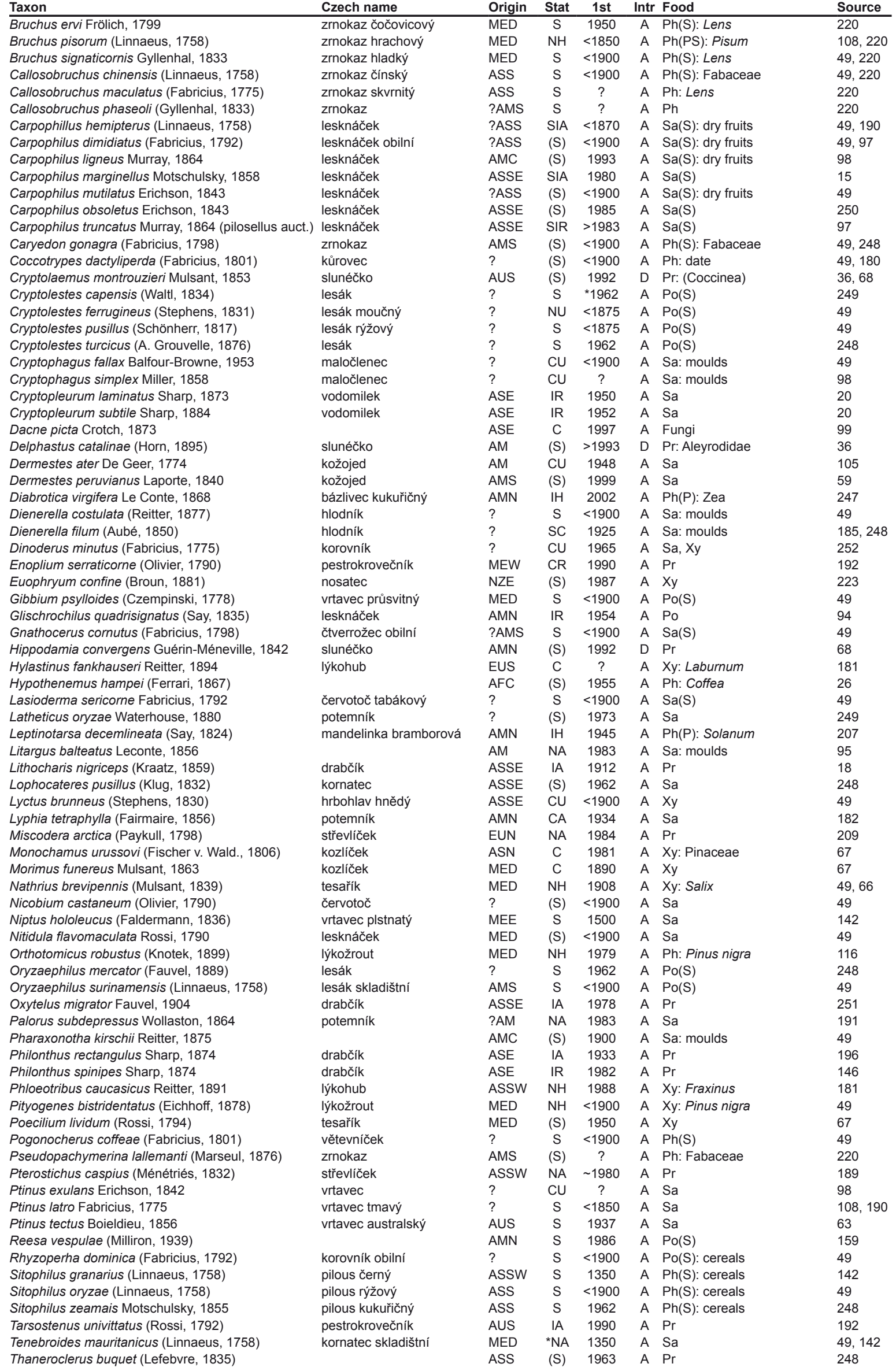




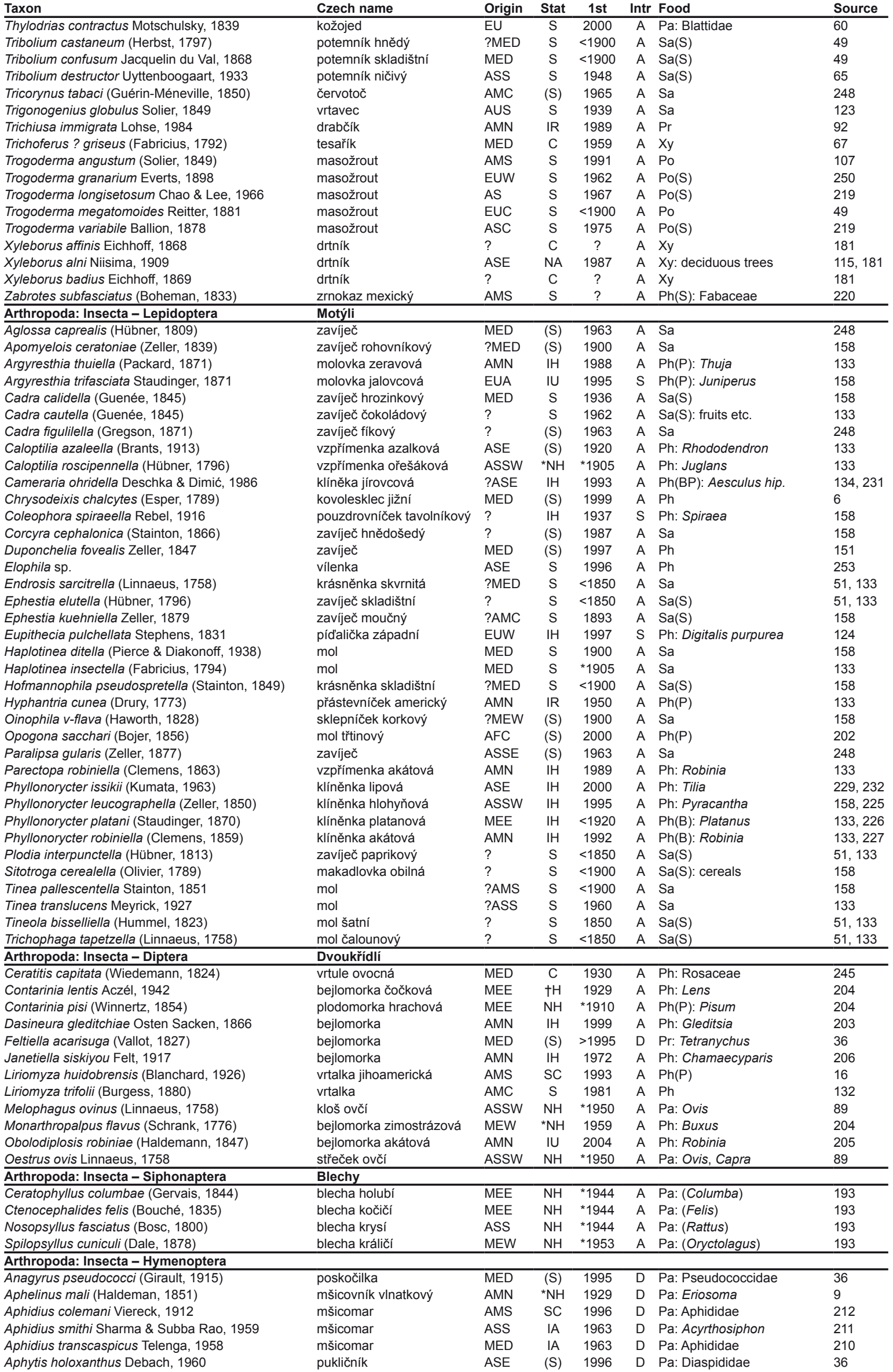




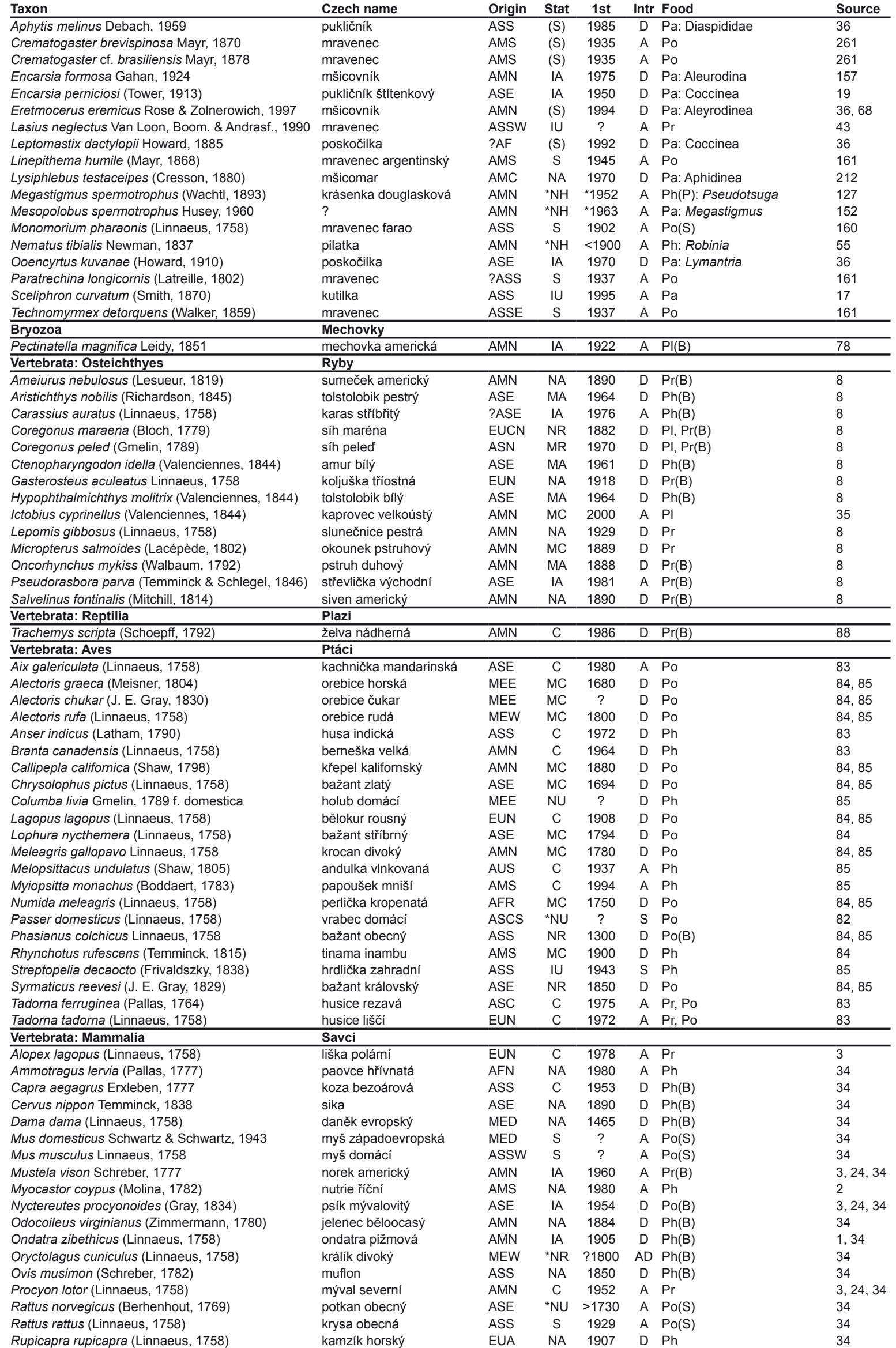


Appendix II. Data sources.

1. ANDĚra, M. \& BenEš, B., 2001: Atlas rozšǐrení savců v České republice IV. Nár. Muz., Praha, $156 \mathrm{pp}$.

2. ANDĚrA, M. \& ČERVENÝ, J., 2003: Lynx, 34: 5-12

3. ANDĚRA, M. \& HANZAL, V., 1996: Atlas rozšírení savcư v České republice II. Nár. Muz., Praha, 86 pp.

4. BÁDr, V., pers. comm., 2005

5. Bajerová, I., 1965: Acta univ. agric. Brno D, 1965: 77-82

6. BÁrtová, E. \& Marek, J., 2000: Zahradnictví, 2(2000): 1, 4

7. BAruš, V., 1965: Fol. Zool., 14: 351-358

8. Baruš, V. \& Oliva, O., 1995: Fauna ČR a SR, sv. 28.

9. BAUDYš, E., 1935: Hospodářská fytopathologie. Díl II. Hubení škůdců živočišných. Brno, $630 \mathrm{pp}$.

10. BAUdyš, E., 1936: Prakt. rádce Milotice n. B. 16 pp.

11. BAUdyš, E., 1948: Acta univ. agric. silvic. Brno, fac. agric., C 43: 1-64

12. BAYER, E., 1914: Moravské hálky (Zoocecidia). Pokorný a spol., Brno, 181 pp.

13. BAYER, E., 1920: Synopsis zoocecidii stredoevropských I. Mor. mus. zemské, Brno, 237 pp.

14. Beran, L., 2002: Sbor. Př́rodověd. klubu v Uh. Hradišti, Suppl. 10, 258 pp.

15. Bílý, S. \& Jelínek, J., 1983: Acta Ent. Bohemoslov., 80: 149-150

16. BitTNER, I. \& ČERnÝ, M., 1994: Ochrana rostlin, 30: 133-139

17. Bogusch, P., 2004: Zool. dny Brno 2004. Sborník abstraktů, 12.-13. února 2004, p. 62

18. ВонÁČ, J., 1985: Acta Ent. Bohemoslov., 82: 360-385, 431-467

19. BouČEK, Z. et al., 1957: Klič zvířeny ČSR, 2: 35-406

20. Boukal, M., 1997: Klapalekiana, 33: 143-149

21. BRyja, J. \& KMENT, P., 2002: Ibid., 38: 1-10

22. Buchar, J. \& RŮŽIČKA, V., 2002: Catalogue of spiders of the Czech Republic. Peres, Praha, 351 pp.

23. ČERnÝ, Z., 1988: Zprávy Čs. společ. ent., 24: 103-104

24. ČERVenÝ, J. et al., 2002: Myslivost, 50(8): 9-13

25. Dejdar, E., 1934: Z. Morph. Ökol. Tiere, 28: 595-691

26. Dirlbek, K. \& Dirlbek, J., 1956: Živa, 4: 63.

27. Dittrich, O., 1974: Práce z oboru zoologie. Klub př́rodověd. Brno, 1974: 13-19

28. Dlabola, J., 1941: Fol. Ent., 4: 140-141

29. Dlabola, J.,1954: Fauna ČSR, sv. 1.

30. Dlabola, J., 1954: Acta Soc. ent. čechosl., 51: 149-155

31. Dlabola, J. (ed.), 1977: Check-list of Czechoslovak Insects I. Národní muzeum, Praha, 160 pp.

32. Drastich, L., 1922: Čas. Čs. společ. ent., 19: 31-62

33. DudA, L., 1892: Catalogus insectorum faunae bohemicae, 1: 1-44
34. Dungel, J. \& Gaisler, J., 2002: Atlas savců České a Slovenské republiky. Academia, Praha, 152 pp.

35. Dungel, J. \& ŘEHÁK, Z., 2005: Atlas ryb, obojživelníků a plazů ČR a SR. Academia, Praha, 184 pp.

36. EPPO, 2005: European and Mediterranean Plant Protection Organisation. http://www.eppo.org

37. Ergens, R. \& Lom, J., 1970: Původci parasitárních nemocí ryb. Academia, Praha, 384 pp.

38. Erhardová, B., 1957: Acta Soc. Zool. Bohemoslov., 21: 148-158

39. Erhardová, B., 1958: Čs. Parasitol., 5(1): 27-103

40. Erhardová, B., 1960: Fol. Zool., 9: 245-248

41. Erhardová-Kotrlá, B., 1971: The occurrence of Fascioloides magna (Bassi, 1875) in Czechoslovakia. Academia, Praha, 155 pp.

42. ERnest, L., 1999: Klapalekiana, 35: 79-80

43. Fauna Europaea, 2005: http//www.faunaeur.org/. Last update 7. 3. 2005

44. Fieber, F. X., 1844: Ent. monogr. Calve, Praha, $138 \mathrm{pp}$.

45. FIEBER, F. X., 1858: Wien. ent. Monatschr., 2: 289-327, 329-347, 388

46. Flasar, I., 1964: Čas. Nár. muz., odd. př́rr., 133: $42-45$

47. Flasarová, M. \& Flasar, I., 1962: Fol. Zool., 11: 71-76

48. Flasarová, M. \& Flasar, I., 1965: Ibid., 14: 251-260

49. Fleischer, A., 1927-1930: Přehled brouků fauny Čs. republiky. Mor. mus. zemské, Brno, 485 pp.

50. Frankenberger, Z., 1944: Věst. Král. čes. společ. nauk, tř. mat-př́r., 1944, odd. 13: 1-28

51. Gartner, A., 1866: Verh. Naturforsch. Ver. Brünn, 4: 48-270

52. Gelnar, M. et al., 1989: Čs. rybnikkářství, 1989: $5-12$

53. Gelnar, M. et al., 1994: Helminthologia, 31: 47-56

54. Gelnar, M. \& Lux, E., 1991: Fol. Parasit., 38: 131-132

55. Gregor, F. \& BAŤA, L., 1942: Sbor. ent. odd. Zem. mus. v Praze, 20, suppl, 88 pp.

56. Groschaft, J., 1956: Čs. Parasitol., 3: 67-74

57. HAJer, J., 1989: Živa, 37: 125

58. Hajer, J. \& Reháková, D., 2003: Bull. Br. Arachnol. Soc., 12: 345-354

59. Háva, J., 1999: Folia Heyrovskyana, 7: 141-150

60. HÁva, J., 2001: Klapalekiana, 37: 123

61. HÁva, J., 2003: Ibid., 39: 128

62. Háva, J., 2004: Ann. Hist.-Natur. Mus. Nat. Hung., 96: 81-95

63. Havelka, J., 1948a: Čas. Čs. společ. ent., 45: $125-126$

64. Havelka J., 1948b: Fol. Ent., 11: 51-72

65. Havelka, J., 1949: Čas. Čs. společ. ent., 46: $155-156$

66. HeyrovskÝ, L., 1919: Ibid., 16: 25-26 
67. Heyrovský, L. \& Sláma, M., 1992: Tesařikovití - Cerambycidae (Coleoptera). Kabourek, Zlín, $368 \mathrm{pp}$.

68. HluchÝ, M., pers. comm., 2005

69. Holá, V. \& Hula, V., 2003: Pavouk, 19: 2

70. Holman, J., 1965: Acta faun. ent. mus. nat. Pragae, 11: 277-284

71. Holman, J., 1971: Acta Ent. Bohemoslov., 68 : 153-166

72. Holman, J., 1991: Acta Univ. Carol., Biol., 35: $19-55$

73. Holman, J., 1995: Fol. fac. sci. natur. Univ. Masaryk. Brun., Biol., 92: 189-200

74. Holman, J. \& Pintera, A., 1977: Check-list of Czechoslovak Insects, I: 101-116

75. HolušA, J., 2000: Čas. Slez. muz. Opava (A), 39: 280

76. HoluŠA, J. et al., 1999: Zool. dny Brno. Abstrakta referátů z konference 4. a 5. 11. 1999, p. 55

77. Horsák, M. et al., 2004: Malacological Newsletter, 22: 141-147

78. Hrabě, S., 1934: Věda př́rodní, 16: 89-92

79. HRABĚ, S., 1954: Klíč zvíreny ČSR, 1: 132-141

80. Hrdý, I. \& Krampl, F., 1977: Acta Ent. Bohemoslov., 74: 286

81. HRDÝ, I. et al., 1979: Ochrana rostlin, 15(52): 259-269

82. Hudec K. (ed.), 1983: Fauna ČSSR, sv. 23.

83. Hudec, K. (ed.), 1994: Fauna ČR a SR, sv. 27.

84. Hudec, K., 2004: Ornit. Mitt., 56: 76-77

85. Hudec, K. \& ŠŤAstnÝ, K. (eds.), 2005: Fauna $\check{C} R, s v .29 / 1,2$.

86. Hudec, V. \& Mácha, S., 1961: Prírodovéd. čas slezský, 22: 303-310

87. Hudečex, J. \& Šunaj, J., 1997: Sbor. Př́rodověd. klubu v Uh. Hradišti, 2: 111-114

88. Chmelík, P. \& Kořínek, M., 1994: Akvárium terárium, 37(3): 37-38

89. Chvála, M. (ed.), 1980: Fauna CSSR, sv. 22.

90. Chytil, J. \& ŘezÁč, M., 2003: Pavouk, 18: 2

91. Ircing, F. \& Plášil, O., 1980: Rybářství, 1980: 223

92. JANÁK, J., 1996: Klapalekiana, 32: 192-193

93. Jarošík, V., 1990: Acta Ent. Bohemoslov., 87: 414-430

94. JelíneK, J., 1984: Ibid., 81: 70-72

95. JELÍNEK, J., 1990a: Ibid., 87: 234-235

96. JeLíneK, J., 1990b: Ibid., 87: 479

97. JelíneK, J., 1990c: Zprav. Západočes. poboč. Čs společ. ent., Plzeň, Suppl., 4: 19-21

98. JelíneK, J., 1993: Check-list of Czechoslovak Insects IV. Fol. Heyrovskyana, Praha, 172 pp.

99. JelíneK, J. \& ŠŤourač, P., 1997: Klapalekiana, 33: 114

100. Jírovec, O., 1948a: Parasitologie pro zvěrolékaře. CAVU Praha, 436 pp.
101. Jírovec, O., 1948b: Parasitologie pro lékaře. Melantrich, Praha, $378 \mathrm{pp}$.

102. Jírovec, O. \& Stecker, J., 1944: Věst. Čs. zool. společ., 9: 48-54

103. JUŘIČKOVÁ, L., 1995: Živa, 43: 30

104. JuŘıČKovÁ, L., 1998: Acta Mus. Reginaehradec. A, 26: 101-172

105. Kalín, V., 1948: Sbor. Př́rodověd. klubu v Pardubicich, 13 pp. +3 pls.

106. Kalík, V., 1986: Acta Ent. Bohemoslov., 83: $155-156$

107. KaLíK, V., 1996: Klapalekiana, 32: 271-273

108. KlikA, J., 1873: Brouci. Kober, Praha, 448 pp.

109. KLIMEŠ, L., 1995: Živa, 43: 76-77

110. KuImeš, L., 2000: Ekológia (Bratislava), 19 (Supl. 3): 125-128

111. KLiMEš, L., pers. comm., 2005.

112. Klimeš, L. \& RoušAr, A., 1998: Arachnol. Mitt., 16: 33-39

113. Kment, P. et al., in press, a: Klapalekiana, 41

114. KMEnt, P. et al., in press, b: Ibid.

115. KnížeK, M., 1988: Acta Ent. Bohemoslov., 85: 396

116. KNí̌ž, M. \& LišKA, J., 1996: Klapalekiana, 32: 76

117. KočÁreK, P. et al., 2005: Blattaria, Mantodea, Orthoptera \& Dermaptera České a Slovenské republiky. Kabourek, Zlín, 348 pp.

118. Kotrlá, B. \& Kotrlý, A., 1973: Fol. Parasit., 20: $377-378$

119. Kotrlá, B. \& Kotrlý, A., 1977: Ibid., 24: 35-40

120. KovAŘ́́, F., 1998: Štírí. Madagaskar, Jihlava, $176 \mathrm{pp}$.

121. Kovař́́, F. (ed.), 2000: Hmyz. Chov, morfologie. Madagaskar, Jihlava, $296 \mathrm{pp}$

122. KRÁL, F., 1943: Zvěrolék. rozpravy, 17(1)

123. KRÁL, J., 1948: Čas Čs. společ. ent. , 45: 127

124. Krampl, F. \& Marek, J., 1999: Sbor. Severočes. muz. - př́r. védy, Liberec, 21: 145-188

125. Kratochvíl, J., 1945: Fol. Ent., 8: 39-67

126. Kratochvíl, J. et al., 1953: Škůdci a choroby pařništ' a sklenikü. ČSAV, Praha, 159 pp.

127. KŘístek, J., 1967: Acta univ. agric. Brno (C), 36(3): 275-286

128. KuČERová, Z., 1992: Acta Ent. Bohemoslov., 89: 315

129. KuČEROVÁ, Z., 1997: Klapalekiana, 33: 116

130. Kuchař, P., 1983: Živa, 31: 23

131. Lang, J., 1959: Acta Soc. Zool. Bohemoslov., 23: 97-122

132. LÁsKA, P., 1982: Záhradníctvo, 7: 218-219

133. LAŠTU゚VKA, Z. (ed.), 1993: Katalog motýli moravskoslezského regionu. AF VŠZ v Brně, 130 pp.

134. LAŠTŮVKA, Z. et al., 1994: Klapalekiana, 30: 197-206

135. Lauterer, P., 1980: Acta Mus. Mor., Sci. natur., 65: $117-140$ 
136. LAUTERER, P., 1982: Ibid., 67: 133-162

137. LaUterer, P., 1984: Ibid., 69: 143-162

138. LAuterer, P., 1993: Ibid., 77(1992): 147-156

139. Lauterer, P., 1995: Ibid., 79(1994): 169-175

140. LAuterer, P., 1996: Ibid., 80: 235-242

141. Lauterer, P., 2002: Plant Protect. Sci., 38(4): $145-148$

142. Lauterer, P., 2004: Zool. dny Brno 2004. Sborník abstraktů, 12.-13. února 2004, p. 86-87

143. Lauterer, P. \& Malenovský, I., 2002: Entomologica Basiliensia, 24: 161-177

144. LišKovÁ, E., 1964: Acta Soc. Zool. Bohemoslov., 28: 305-311

145. LožEK, V., 1956: Kličč československých měkkýšů SAV, Bratislava, $437 \mathrm{pp}$.

146. MácA, J., 1984: Acta Ent. Bohemoslov., 81: 398

147. MÁcA, J., 1991: Acta Soc. Zool. Bohemoslov., 55: $1-11$

148. Мácha, S., 1971: Acta Mus. Silesiae, Ser. A, 20 : 121-134

149. MÁcha, S., 1988: Ibid., 37: 63-64

150. МАСнÁČEK, P., 1977: Scripta fac. sci. natur. UJEP Brunensis, Biol., 2(7): 71-86

151. Marek, J. \& BÁrtová, E., 1998: Plant. Protect. Sci., 34: 151-152

152. Mentberger, J., 1964: Fol. Zool., 13: 185-186

153. Miller, F., 1956: Zemédělská entomologie. ČSAV, Praha, 1057 pp.

154. Moravec, F., 1992: Fol. Parasitol., 39: 247-248

155. Moravec, F., 2001: Checklist of the metazoan parasites of fishes of the Czech Republic and the Slovak Republic (1873-2000). Academia, Praha, $172 \mathrm{pp}$.

156. Mrciak, M. \& Rosický, B., 1956: Fol. Zool., 5: 143-148

157. NavrÁtilovÁ, M., 1999: Ověrování biologických metod ochrany rostlin ve skleníkových kulturách. Dis. Práce AF MZLU Brno (MS), 151 pp.

158. NovÁK, I. \& LišKA, J. (eds), 1997: Klapalekiana, 33 (Suppl.): 1-159

159. NovÁk, I. \& Verner, P., 1990: Acta Ent. Bohemoslov., 87: 479

160. NovÁk, V., 1942: Čas. Čsl. společ. ent., 39: 135-136

161. NovÁK, V., 1947: Ibid., 44: 144-146

162. Obenberger, J., 1926: Rovnokřidlý hmyz (Orthoptera a Dermaptera) Republiky Československé. ČAVU, Praha, 234 pp.

163. Obenberger, J., 1929: Vesmír, 7: 202-203

164. Овв, S., 1948: Spisy prír. fak. Masaryk. univ., M2, 306, $108 \mathrm{pp}$.

165. Oвв, S., 1954: Přir. sbor. Ostravského kraje, 15: 269-283

166. Овв, S., 1978: Acta Ent. Bohemoslov., 75: 226-242
167. OndračKovÁ, M. \& JuRAJdA, P., 2000: Helminthologia, 37: 174

168. ONDRAČKOVÁ, M. et al., 2004: Ibid., 41: 139-145

169. PAŠEK, V., 1954: Vošky našich lesných drevín. Bratislava, $322 \mathrm{pp}$.

170. Pelikán, J., 1952: Fol. Zool. Ent., 1(15): 185-194

171. Pelikán, J., 1957: Fol. Zool., 6: 52-56

172. Pelikán, J., 1965: Acta Ent. Bohemoslov., 62: 98-104

173. Pelikán, J., 1977: Acta faun. ent. mus. nat. Pragae, 15, Suppl. 4: 55-59

174. PelikÁn, J., 1989: Ochrana rostlin, 25(4): 271-278

175. PelikÁn, J., 1990: Acta Ent. Bohemoslov., 87: 232-237

176. PelikÁn, J., 1991: Ochrana rostlin, 27(3-4): 287-291

177. Pelikán, J., 1998: Ibid., 34(1): 39-42

178. Pelikán, J. \& Schliephake, G., 1994: Ent. Ztschr., 104: 181-185

179. Petrusek, A., 2002: Acta Soc. Zool. Bohem., 66: 213-220

180. Pfeffer, A., 1989: Kůrovcovití Scolytidae a jádrohlodovití Platypodidae. Academia, Praha, $139 \mathrm{pp}$.

181. PfefFer, A. \& KnížeK, M., 1989: Lesnická práce, 68: $311-312$

182. PickA, J., 1978: Zprávy Čs. společ. ent., Suppl., $53 \mathrm{pp}$.

183. PInTERA, A., 1959: Klíč zviřreny ČSR, 3: 471-525

184. PižL, V., 2002: Sbor. Př́rodověd. klubu v Uh. Hradišti, Suppl. 9, 154 pp.

185. PrůdeK, P., 1997: Klapalekiana, 33: 249-250

186. PřibyslavskÝ, J. et al., 1965: Acta Soc. Zool. Bohemoslov., 29: 5-8

187. PřIdAL, A., pers. comm., 2005

188. Pucholt, R., 1985: Acta Ent. Bohemoslov., 82: 59-64

189. Pulpán, J. \& Hůrka, K., 1984: Zprav. Západočes. pob. Čs. společ. ent. v Plzni, 1(Suppl.): $1-28$

190. ReitTer, E., 1870: Verh. Naturforsch. Ver. Brünn., 8(2): $1-195$

191. RičL, P., 1985: Acta Ent. Bohemoslov., 82: 152

192. RoLČík, J., pers. comm., 2005

193. RosickÝ, B., 1957: Fauna ČSR, sv. 10.

194. RosickÝ, B., 1959: Klíč zviřeny ČSR, 3: 271-276

195. RosickÝ, B. \& DANiel, M. (eds), 1989: Lékařská entomologie a životní prostředí. Academia, Praha, $439 \mathrm{pp}$.

196. Roubal, J., 1933: Čas. Čs. společ. ent., 30: 152

197. RouŠAR, A., in KLIMEŠ (2000)

198. RŮŽIČKA, V., 1995: Eur. J. Ent., 92: 723-726

199. RŮŽıčKA, Z., 1977: Živa, 25: 224

200. Ryšavý, B. \& Erhardová, B., 1953: Parasiti ovcí. ČSAV, Praha, 191 pp. 
201. Sabová, M. et al., 1993: Parazitické nematódy - škodcovia rastlín. VSAV, Bratislava, 143 pp.

202. SiteK, J., 2003: Klapalekiana, 39(1-3): 137-138

203. SkuhravÁ, M., 2004: Fol. fac. sci. natur. Univ. Masaryk. Brun., Biol., 109: 324-331

204. Skuhravá, M. \& SkuhravÝ, V., 1960: Bejlomorky. ČAZV, SZN Praha, 273 pp.

205. SkuhravÁ, M. \& SkuhravÝ, V., 2004: Lesnická práce, 83: 520

206. SkuhravÝ, V. \& SkuhravÁ, M., 1998: Bejlomorky lesnich stromů a keřu. Matice lesnická, Písek, $176 \mathrm{pp}$.

207. SlabÝ, V. et al., 1950: Ochrana rostlin, 23(3): 230-234

208. Smetana, A., 1965: Acta rer. natur. Mus. nat Slov. Bratislava, 11: 30-83

209. Stanovský, J. et al., 1986: Ent. zprav., Ent. klub Ostrava-Poruba, 16: 41-81

210. Starý, P., 1965: Ann. Soc. Ent. Fr., N.S., 1: 177-180

211. StARÝ, P., 1966: Aphid parasites of Czechoslovakia. Academia, Praha, 242 pp.

212. STARÝ, P., pers. comm., 2005

213. Starý, P. \& Havelka, J., 1990: Ochrana rostlin, 26(1): 53-58

214. Sтенlík, J. L., 1962: Acta Mus. Moraviae, Sci. natur., 47: 125-133

215. STEHLík, J. L., 1997: Ibid., 81: 299-306

216. Stehlík, J. L. \& Hradil, K., 2000: Ibid., 85: 351-353

217. Stehlík, J. L. \& VAVŘínOvÁ, I., 1997: Ibid., 81(1996): 231-298

218. Stejskal, V., 1993: Anz. Schädlingsknd., 66: 150-151

219. Stejskal V. et al., 2005: Plant Protect. Sci., 41: $42-45$

220. STReJČEK, J., 1990: Brouci čeledí Bruchidae, Urodontidae a Anthribidae. Academia, Praha, 88 pp.

221. StreJČEK, J., 1993: Klapalekiana, 29: 169-171

222. STREJČEK, J., 2001: Katalog brouků (Coleoptera) Prahy, sv. 2. Praha, 138 pp.

223. StRejČek, J., pers.comm., 2005

224. SuKop, I. \& SEDLÁK, E., 1999: Fol. fac. sci. natur. Univ. Masaryk. Brun., Biol., 101: 163-165

225. ŠEfrová, H., 1999: Acta univ. agric. silvic. Mendel. Brun., 47(3): 57-63

226. Š EFrovÁ, H., 2001: Ibid., 49(5): 71-75

227. ŠEFrovÁ, H., 2002a: Ibid., 50(3): 7-12

228. ŠEFrovÁ, H., 2002b: Ibid., 50(3): 99-104

229. ŠEFrovÁ, H., 2003: Ekológia (Bratislava), 22: $132-142$

230. Šefrová, H., unpubl., Brno, 25.v.2005

231. Šefrová, H. \& LAŠTŮVKA, Z., 2001: Ent. Ztschr. Frankfurt, 111: 194-198

232. ŠEFrovÁ, H. et al., 2000: Klapalekiana, 36: 326

233. ŠILHAVÝ, V., 1956: Fauna ČSR sv. 7.
234. ŠKoríkovÁ, B. et al., 1996: Helminthologia, 33 : 168-169

235. ŠpryŇAr, P., 2005: Plant Protect. Sci., 41: 38-41

236. ŠPryŇAR, P. \& ERNEST, L., 2001: Klapalekiana, 37: $125-127$

237. Šteflová-Leiská, M., 1957: Čs. Parasitol., 4: 337-350

238. ŠTusÁK, J. M., 1981: Sbor. VŠZ v Praze, fak. agron., řada $B, 33: 49-58$

239. Tenora, F., 1956: Acta univ. agric. silvic. Brno (A), 1956: 37-50

240. Tenora, F., 1964: Fol. Zool., 13: 88-89

241. TenOra, F., pers. comm. 2005

242. Tenora, F. \& Baruš, V., 1957: Fol. Zool., 6: 341-357

243. Tenora, F. et al., 1974: Acta Soc. Zool. Bohemoslov., 38: 71-75

244. Tenora, F. \& StaněK, M., 1990: Helminthologia, 27: 73-77

245. Tı́HÁ, H., 1967: Ochrana rostlin, 3: 307-308

246. UličNÝ, J., 1892-5: Měkkýši čeští. Klub přírodověd. Praha, 208 pp.

247. Vahala, O. \& BezděK, J. 2002: Plant Protect. Sci., 38: 114

248. Verner, P. et al., 1965: Výzkum fauny závodů potravinářského průmyslu. Závěrečná zpráva, dílči úkol č. 1. VÚPP, Praha, 556 pp.

249. VERNER, P. et al., 1975: Výzkum importovaných škůdců a metod jejich hubení na dovážených obilovinách včetně rýže. Záv. Zpráva. VÚPP, Praha, $146 \mathrm{pp}$.

250. Verner, P. et al., 1986: Fauna skladištních škůdců zemédělsko-potravinářského komplexu. Záv. Zpráva. VÚPP, Praha, 105 pp.

251. Vogel, J., 1981: Acta Ent. Bohemoslov., 78: 324

252. Volák, J., 1965: Zprávy Čs. společ. ent., 1(4): 18-19

253. VRABEC, V. et al., in prep.

254. VysloužIl, L., 1986: Acta Ent. Bohemoslov., 83: 154

255. Zahradní, J., 1959: Acta faun. ent. mus. nat. Pragae, suppl. 1, 70 pp.

256. ZAHRADNí, J., 1961: Ibid., 7: 61-80

257. ZAHRADNíK, J., 1965: Ibid., 11: 303-30

258. Zahradník, J., 1990: Acta Univ. Carol. Biol., 33(1989): 455-457

259. ZAHRADNÍK, J., 1990: Ibid., 34: 1-160

260. ZAJÍČEK, D., 1955: Acta univ. agric. silvic., Brno (B), 1955: 29-38

261. ZÁlesKÝ, M., 1937: Záhorská kronika, 20(2): 1

262. ZÁleský, M., 1940: Př́roda, 33: 133-136

263. Zapletal, M., 1960: Fol. Zool., 9: 47-58

264. Zounhe, M. et al., 2003: Plant Dis., 87: 9

265. Žofrová, M. et al., 2002: Acta Soc. Zool. Bohem., 66: 221-230 


\section{SOUHRN}

Katalog druhů živočichů cizího původu v České republice

Z území České republiky je známo asi 34000 druhů mnohobuněčných živočichů (Metazoa). Druhové složení fauny je kromě př́rodních faktorů zásadně ovlivněno nejrưznějšími aktivitami člověka trvajícími od počátku neolitu, tj. asi 6700 let. Zvláště v posledních několika staletích člověk přímo nebo nepř́mo ovlivnil výskyt a šiření mnoha živočišných druhů. Záměrně byly introdukovány druhy s předpokládaným užitkem (domácí zviřata, lovná zvěř a ryby, predátoři a parazité využivaní v ochraně rostlin), druhy projevující se negativně byly obvykle zavlečeny nechtěně (škůdci materiálů a rostlin, parazité, druhy narušující místní biodiverzitu). Práce je prvním pokusem předložit soupis cizích a invazních druhů živočichů v České republice. Katalog obsahuje informace o původu jednotlivých druhů, datum prvního výskytu (pozorování), způsob introdukce (náhodný - A, záměrný - D, spontánní - S), invazní statut druhu (eusynantropní - S, př́ležitostný volný výskyt - C, zdomácnělý neinvazní - N, zdomácnělý postinvazní - *N, invazní - I), potravní nároky a možné vlivy. Kromě vědeckých názvů jsou uvedena i česká jména druhů $z$ důvodu předpokládaného použití katalogu především v České republice. Katalog zahrnuje celkem 595 druhů cizího původu, tj. 1,8 \% známé České fauny, z toho 22 měkkýšů $(8,8 \%$ místní fauny), 451 členovců (1,5\%), 383 druhů hmyzu (1,4 \%) a 55 obratlovců $(9,2 \%)$. Ze zaregistrovaných druhů je 248 (41,8\%) výskytem omezeno jen na uzavřené vytápěné prostory, 60 druhů $(10,1$ \%) přežívá ve vnějším prostředí jen prríležitostně a krátkodobě a 287 druhů $(48,2 \%)$ ve vnějším prostředí zdomácnělo (naturalizované druhy). Z nich se 105 (17,7 \% všech cizích) v novém území nešǐrí (neinvazní druhy), invaze 69 druhů (11,6 \%) proběhla v minulosti (postinvazní druhy) a 113 druhů (19 $\%)$ je invazních. Nepůvodních 84 druhů (14,1 \%) jsou parazité živočichů, 65 druhů $(10,9 \%)$ patří mezi skladištní škůdce, 53 druhů $(8,9 \%$ ) škodí na rostlinách ve vytápěných prostorech (zejména ve sklenících), 28 druhů (4,7 \%) škodí v zemědělství a lesnictví a 39 druhů (6,6 \%) může mít vliv na místní biodiverzitu. Zdomácnělé cizí druhy pocházejí nejčastěji ze Severní Ameriky (70;24,4 \%), Mediteránu (61; 21,3 \%), z Východní (44; 15,4 \%), Střední a jihozápadní (43; 15 \%) a Jižní až jihovýchodní Asie $(30 ; 10,5 \%)$.

živočichové, cizí původ, invazní druhy, Česká republika

\section{ACKNOWLEDGEMENTS}

We are obliged for consultations, various amendments, and comments on the list of the alien species in the respective taxa to the following zoologists: V. Bádr (Mallophaga, Anoplura, Siphonaptera, parasitic Acarina, general comments and discussions), V. Baruš (Trematoda, Cestoda, Nematoda), J. Bezděk (Coleoptera, important literature and data sources), J. Bryja (Heteroptera), M. Černý (Diptera: Agromyzidae), F. Gregor (Diptera, general discussions), J. Hajer (Araneida), J. Háva (Coleoptera: Dermestidae), J. Havelka (Aphidinea), M. Hluchý (species used in biological control), M. Horsák (Mollusca), K. Hudec (Aves and other Vertebrata, general discussions), V. Hula (Araneida, Blattodea, etc.), J. Jelínek (Coleoptera), P. Jelínek (Coleoptera), J. Kaláb (Coleoptera: Carabidae), L. Klimeš (Opiliones), P. Kment (Heteroptera), J. Kolibáč (Coleoptera), P. Koubek (Mammalia), P. Lauterer (Psyllinea, Auchenorrhyncha), J. Liška (Lepidoptera, Hymenoptera), I. Malenovský (Psyllinea, Auchenorrhyncha, etc.), J. Pelikán (Thysanoptera), P. Průdek (Coleoptera), J. Rolč́́k (Coleoptera: Cleridae), R. Rozkošný (Diptera), M. Skuhravá (Diptera: Cecidomyiidae), P. Spurný (Osteichthyes), P. Starý (Hymenopteran parasitoids, Aphidinea), J. Strejček (Coleoptera: Chrysomelidae, Curculionidae), I. Sukop (aquatic Crustacea), H. Šejnohová (Aves, etc.), F. Št’áhlavský (Arachnida: Pseudoscorpionida), F. Tenora (Trematoda, Cestoda, Nematoda), I. H. Tuf (terrestrial Isopoda), O. Vahala (Heteroptera), M. Zacharda (Acarina used in biological control) and J. Zahradník (Coccinea); V. Grulich and L. Úradníček offered various data on the alien plant species and Z. Pažourková accommodated us with the loan of the important literature. R. Obrtel has kindly translated the text into the English language.

This study was supported by the Research Grant from MSM: 432100001. 


\section{REFERENCES}

AGASSIZ, D. J. L.: Invasions of Lepidoptera into the British Isles, p. 9-36. In: Emmet, A. M. (ed.), The moths and butterflies of Great Britain and Ireland, vol. 3. Harley Books, Colchester, 1996, 452 pp.

BARUŠ, V., OLIVA, O. (eds): Mihulovci Petromyzontes a ryby Osteichthyes. Fauna $\check{C} R$ a SR, sv. 28/ 1, 2. Academia, Praha, 1995, $624+703$ pp.

ČERNÝ, V.: First case of introduction of the tick Rhipicephalus sanguineus to Czechoslovakia. Fol. $\mathrm{Pa}$ rasit., 1985, 32: 162

DAVIS, M. A., THOMPSON, K.: Invasion terminology: should ecologists define their terms differently than others? No, not if we want to be of any help! Bull. Ecol. Soc. Am., 2001, 82: 206

ELTON, C. S.: The ecology of invasion by animals and plants. Methuen, London, 1958, 181 pp.

ERHARDOVÁ-KOTRLÁ, B.: The occurence (sic) of Fascioloides magna (Bassi, 1875) in Czechoslovakia. Academia, Praha, 1971, 155 pp.

ESSL, F., RABITSCH, W.: Neobiota in Österreich. Umweltbundesamt, Wien, 2002, 432 pp.

FRANK, S.: Acclimatization experiments with amur snake head, Ophiocephalus argus Warpachowskii Berg, 1909 in Czechoslovakia. Acta Soc. Zool. Bohemoslov., 1970, 34: 277-283

GEITER, O., HOMMA, S., KINZELBACH, R.: Bestandsaufnahme und Bewertung von Neozoen in Deutschland. Texte des Umweltbundesamtes 25/02, Berlin, 2002, 262 pp.

KOWARIK I.: Biologische Invasionen - Neophyten und Neozoen in Mitteleuropa. Ulmer, Stuttgart, 2003, $382 \mathrm{pp}$

KRÜGER, L.: Insektenwanderungen zwischen Deutschland und den Vereinigten Staaten von Nordamerika und ihre wirtschaftliche Bedeutung. Friedländer, Berlin, 1899, 174 pp.

LAUTERER, P.: Subfosilie členovců a žížal v České republice, p. 86-87. In: Bryja J., Zukal J. (eds), Zoologické dny Brno 2003. Sbornik abstraktio z konference 12.-13. února 2004, 2004, 233 pp.

MANCHESTER, S. J., BULLOCK, J. M.: The impacts of non-native species on UK biodiversity and the effectiveness of control. J. Appl. Ecol., 2000, 37: $845-864$

PIMENTEL, D. (ed.): Biological invasions. Economic and environmental costs of alien plant, ani- mal, and microbe species. CRC Press, Boca Raton, 2002, 369 pp.

PYŠEK, P.: Past and future of prediction in plant invasions: a field test by time. Diversity and Distributions, 2001, 7: 145-151

PYŠEK, P., SÁDLO, J., MANDÁK, B.: Catalogue of alien plants of the Czech Republic. Preslia, Praha, 2002, 74: 97-186

RICHARDSON, D. M., PYŠEK, P.: What is an invasive species? http://www.cabicompendium.org/cpc/ library/InvasivePlants/1\%20intro.htm, 2004

RICHARDSON, D. M., PYŠEK, P., REJMÁNEK, M., BARBOUR, M. G., PANETTA, F. D., WEST, C. J.: Naturalization and invasion of alien plants: concepts and definitions. Diversity and Distributions, 2000, 6: 93-107

STEHLÍK, J. L., HRADIL, K.: Arocatus longiceps Stål in the Czech Republic too (Lygaeidae, Heteroptera). Acta Mus. Moraviae, Sci. Biol., 2000, 85: 351-353

STEJSKAL, V., KUČEROVÁ, Z.: Survey of stored-product pests in rice imported from Vietnam. Ochrana Rostlin, 1993, 29: 187-191

ŠEFROVÁ, H.: Phyllonorycter platani (Staudinger) - a review of its dispersal history in Europe (Lepidoptera, Gracillariidae). Acta Univ. Agric. et Silvic. Mendel. Brun., 2001, 49(5): 71-75

ŠEFROVÁ, H.: Invasions of Lithocolletinae species in Europe - causes, kinds, limits and ecological impact (Lepidoptera, Gracillariidae). Ekológia (Bratislava), 2003, 22: 132-142

TENORA, F.: Příspěvek k poznání helmintofauny ondatry pižmové (Ondatra zibethica L.) v ČSR. Acta Univ. Agric. Silvic. Brno (A), 1956: 37-50

WEBB, D. A.: What are the criteria for presuming native status? Watsonia, 1985, 15: 231-236

WHITE, P. C. L., HARRIS, S.: Economic and environmental costs of alien vertebrate species in Britain, p. 113-149. In: Pimentel, D., Biological invasions. Economic and environmental costs of alien plant, animal, and microbe species. CRC Press, Boca Raton, 2002, 369 pp.

WILIAMSON, M.: Alien plants in the British Isles, $p$. 91-112. In: Pimentel, D., Biological invasions. Economic and environmental costs of alien plant, animal, and microbe species. CRC Press, Boca Raton, 2002, 369 pp.

\section{Address}

Ing. Hana Šefrová, Ph.D., Ústav pěstování, šlechtění rostlin a rostlinolékařství, Prof. RNDr. Zdeněk Laštůvka, CSc., Ústav zoologie, rybářství, hydrobiologie a včelařství, Mendelova zemědělská a lesnická univerzita v Brně, Zemědělská 1, 61300 Brno, Česká republika 